\title{
An improvement of Laguerre computational scheme for solv- ing nonlinear age-structured population models
}

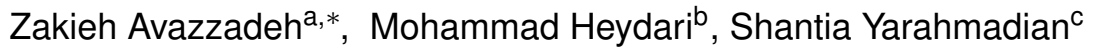 \\ a School of Mathematical Sciences, Nanjing Normal University, Nanjing 210023, China. \\ ${ }^{b}$ Department of Mathematics, Yazd University, P. O. Box: 89195-741, Yazd, Iran. \\ ${ }^{c}$ Mississippi State University, MS 39762, United States.
}

\begin{abstract}
In this paper, we simultaneously implement two kinds of orthogonal polynomials for solving a nonlinear age-structured population model. This non-classic type of partial differential equation is typically defined in large domains that makes finding an accurate solution by common techniques to be difficult. The presented method namely modified generalized LaguerreChebyshev (MGLC), which is based on the modified generalized Laguerre functions and Chebyshev orthogonal polynomials provides the spectral accuracy. The theoretical and experimental analysis of the scheme reliability verifies the validity of the proposed method in large domains.
\end{abstract}

Keywords: Nonlinear age-structured population model, generalized Laguerre functions, modified generalized Laguerre functions, orthogonal polynomials, error analysis.

2010 MSC: 35Q92, 92D25, 74S25, 94A11.

(C)2019 All rights reserved.

\section{Introduction}

Individuals in a structured population are distinguished by age, size, maturity or some other individual physical characteristics. In modeling population dynamics, the main assumption is that the structure of the population with respect to these individual physical characteristics at a given time, and possibly some environmental inputs in time, completely determines the dynamics behavior of the population. Mathematical models describing this evolution have attracted a considerable amount of interest among researchers as a tool for modelling the interactions of different population communities in diverse fields such as demography, epidemiology, ecology, cell kinetics, tumor growth, etc. [2].

In this paper, we study the following form of the nonlinear age-structured population model, which is written as

$$
\frac{\partial u(t, x)}{\partial t}+\frac{\partial u(t, x)}{\partial x}=-\left[d_{1}(x)+d_{2}(x) u(t)\right] u(t, x), \quad 0 \leqslant t, \quad 0 \leqslant x<A,
$$

\footnotetext{
*Corresponding author

Email address: z.avazzadeh@njnu.edu.cn (Zakieh Avazzadeh)

doi: $10.22436 /$ jmcs.019.04.07
}

Received: 2017-12-01 Revised: 2019-05-22 Accepted: 2019-06-15 


$$
\begin{aligned}
u(0, x) & =u_{0}(x), \quad 0 \leqslant x<A, \\
u(t, 0) & =\int_{a}^{A}\left[b_{1}(s)-b_{2}(s) u(t)\right] u(t, s) d s, \quad 0 \leqslant t, \\
u(t) & =\int_{0}^{A} u(t, s) d s, \quad 0 \leqslant t,
\end{aligned}
$$

where $t, x$ denote time and age, respectively, $U(t)$ is the total population at time $t, u(t, x)$ is the agespecific density of individuals of age $x$ at time $t$, which means that $\int_{a}^{a+\Delta a} u(t, s) d$ s counts the number of individuals that have age between $a$ and $a+\Delta a$ at time $t, d_{1}(x)$ is the natural death rate (without considering the competition), $d_{2}(x) U(t)$ is the increase of death rate considering competition, $b_{1}(t)$ is the natural fertility rate (without considering the competition), $b_{2}(x) U(t)$ is the decrease of fertility rate considering the competition, a denotes the lowest bearable age, and $A$ is the maximum age that an individual of the population may reach. In fact, this is the particular form of the following general model $[17,35]$

$$
\begin{aligned}
\frac{\partial u(t, x)}{\partial t}+\frac{\partial u(t, x)}{\partial x} & =-\mu\left(x, I_{\mu}(t), t\right) u(t, x), \quad 0 \leqslant t, \quad 0 \leqslant x<A, \\
u(0, x) & =u_{0}(x), \quad 0 \leqslant x<A, \\
u(t, 0) & =\int_{a}^{A} \beta\left(s, I_{\beta}(t), t\right) u(t, s) d s, \quad 0 \leqslant t,
\end{aligned}
$$

when $\beta$ and $\mu$ denote fertility and death rate, respectively, $I_{\mu}(t)=\int_{a}^{A} \gamma_{\mu}(x) u(t, x) d x$ and $I_{\beta}(t)=$ $\int_{a}^{A} \gamma_{\beta}(x) u(t, x) d x$ and $\gamma_{\mu}(x), \gamma_{\beta}(x)$ are weight functions; $\gamma_{\mu}(x)=\gamma_{\beta}(x) \equiv 1, \mu(x, z, t)=d_{1}(x)+d_{2}(x) z$ and $\beta(x, z, t)=b_{1}(x)-b_{2}(x) z$.

Since aspects of this age-structured population model such as nonlinearity and integral boundary condition which cause difficulty and complexity in finding the solution, various alternative techniques have been developed to obtain analytical and numerical solutions of this model in the literature. A spline algorithm for solving the nonlinear age-structured population model is employed in [26]. Kim and Park developed an upwind scheme for this model [31]. Abia et al. [2] applied the forward difference schemes based on the Runge-Kutta method to this model. Cui and Chen [17] used the reproducing kernel method for the nonlinear age-structured population model. Krzyzanowski et al. [33] obtained a piecewise regular solution of nonlinear age-structured population model by using the discontinuous Galerkin method. Xiuying $\mathrm{Li}$ [35] provided an analytic algorithm for the nonlinear age-structured population model by using the variational iteration method. Hosseini et al. [25] employed the variational iteration method coupled with an auxiliary parameter to solve the nonlinear age-structured population model. In [45], a numerical method for solving the nonlinear age-structured population models is presented, which is based on Bernstein polynomials approximation. A comparison study between homotopy analysis method and optimal homotopy asymptotic method for the solution of nonlinear age-structured population models has been presented by Ghoreishi et al. [21]. For more information on the mathematical modeling and solution of this model, we refer the interested reader to $[1,3-5,7,8,11,18,30,32,46]$ and references therein.

As mentioned in [5], two of the main objectives driving the need for numerical methods are, first, the need to make projections about population growth for the future, usually for periods of 10-50 years. Secondly, there is the theoretical interest in long-term simulations for the purpose of analyzing trends under different scenarios. This is an important aspect of population models in theoretical biology.

Since the model is popular for long term individual physical characteristics at a given time (short or long period of time), the validity and convergence of the method should be guaranteed for large intervals. In this study, we propose the method based on implementation of Laguerre orthogonal polynomials, which leads toward the spectral method in approximating function defined in large domain $[15,16,40,41]$. This scheme has been hired several times to study some difficult systems in Mechanics, Physics, etc. $[10,22,24,43,44]$. This method is privileged by an exponential rate of convergence. The other advantage 
of this method is simplicity in unifying the boundary condition and the equation under study. We remind that, age-structured population model is associated with integral boundary condition which causes very complicated computations. Actually, in some methods such as FEM, FDM, etc., dealing with boundary conditions is a costly challenge but MGLC method is flexible with respect to boundary conditions.

The paper is organized in the following way. In Section 2, Laguerre and Chebyshev orthogonal polynomials are briefly described. In Section 3, we approximate the solution via two different orthogonal polynomials simultaneously applied in two different directions. This section explains the algorithm for the reduction of the partial integro-differential equation to an algebraic system of equations due to generalized Laguerre functions and Chebyshev polynomials. In Section 4, some numerical examples are demonstrated, which confirm the accuracy and applicability of the method and furthermore, error analysis and reliability of the proposed method are discussed. The last section includes some other features of the modified generalized Laguerre-Chebyshev (MGLC), conclusion and further ideas for future work.

\section{Mathematical preliminaries and notations}

In this section, we briefly review the Chebyshev polynomials, generalized Laguerre polynomials, modified generalized Laguerre functions and Gauss-Legendre integration rule. For more details see $[6,15,16,28,29,34,36-38,41]$.

\subsection{Chebyshev polynomials}

Chebyshev polynomials are the well known family of orthogonal polynomials that have many applications $[15,16]$. The Chebyshev polynomial of degree $n$ on $[-1,1]$ is defined by the formula

$$
\mathrm{T}_{\mathrm{n}}(\mathrm{t})=\cos (\mathrm{n} \arccos (\mathrm{t})) .
$$

Clearly, $\left|T_{n}(t)\right| \leqslant 1$ for $t \in[-1,1], T_{0}(t)=1, T_{1}(t)=t$ and, using elementary trigonometric identities, is satisfied the following recurrence formula

$$
T_{n+1}(t)=2 t T_{n}(t)-T_{n-1}(t), \quad n=1,2, \ldots
$$

This is an orthogonal system with the weight function $w(t)=\frac{1}{\sqrt{1-t^{2}}}$ and orthogonality property

$$
\int_{-1}^{1} \frac{T_{n}(t) T_{m}(t)}{\sqrt{1-t^{2}}} d t=\frac{\pi}{2} c_{n} \delta_{n, m}
$$

where $c_{0}=2, c_{n}=1, n \geqslant 1$ and $\delta_{n, m}$ is the Kronecker delta function.

A function $f(t) \in L_{w}^{2}(-1,1)$, may be expressed in terms of Chebyshev polynomials as

$$
f(t)=\sum_{j=0}^{\infty} f_{j} T_{j}(t),
$$

where the coefficients $f_{j}$ are given by

$$
f_{j}=\frac{2}{\pi c_{j}}\left\langle f(t), T_{j}(t)\right\rangle_{w}, \quad j=0,1,2, \ldots
$$

Here, $\langle., .\rangle_{w}$ is the inner product of $\mathrm{L}_{w}^{2}(-1,1)$.

Theorem 2.1 ([16]). Let $f(t) \in H_{w}^{k}(-1,1)$ and $f_{N}(t)=\sum_{j=0}^{N} f_{j} T_{j}(t)$ be the best approximation polynomial of $\mathrm{f}(\mathrm{t})$ in $\mathrm{L}_{w}^{2}$-norm, then

$$
\left\|f(t)-f_{N}(t)\right\|_{L_{w}^{2}[-1,1]} \leqslant C_{0} N^{-k}\|f(t)\|_{H_{w}^{k}(-1,1)},
$$

where $\mathrm{C}_{0}$ is a positive constant, which depends on selected norm and independent with $\mathrm{f}(\mathrm{t})$ and $\mathrm{N}$. 
For practical use of Chebyshev polynomials on $[0, \mathrm{~T}]$, it is necessary to shift the defining domain by the following variable substitution

$$
\mathrm{t}=2\left(\frac{x}{\mathrm{~T}}\right)-1
$$

Denoting the shifted Chebyshev polynomials $T_{n}\left(2\left(\frac{x}{T}\right)-1\right)$ by $T_{n}^{*}(x)$, then these polynomials can be obtained by using the following recurrence formula

$$
\mathrm{T}_{\mathrm{n}+1}^{*}(\mathrm{x})=\left(4\left(\frac{x}{\mathrm{~T}}\right)-2\right) \mathrm{T}_{\mathrm{n}}^{*}(\mathrm{x})-\mathrm{T}_{\mathrm{n}-1}^{*}(\mathrm{x}), \quad \mathrm{n}=1,2, \ldots,
$$

where $T_{0}^{*}(x)=1$ and $T_{1}^{*}(x)=2\left(\frac{x}{T}\right)-1$. The orthogonality condition is written as

$$
\int_{0}^{T} T_{n}^{*}(x) T_{m}^{*}(x) w^{*}(x) d x=\frac{\pi}{2} c_{n} \delta_{n, m}
$$

where $w^{*}(x)=\frac{1}{\sqrt{T x-x^{2}}}$.

Corollary 2.2. Let $f\left(\frac{T}{2}(x+1)\right) \in H_{w}^{k}(-1,1), T>0$ and $f_{N}^{*}(x)=\sum_{j=0}^{N} f_{j}^{*} T_{j}^{*}(x)$ be the best approximation polynomial of $\mathrm{f}(\mathrm{x})$ in $\mathrm{L}_{w^{*}}^{2}$ norm, then

$$
\left\|f(x)-f_{N}^{*}(x)\right\|_{L_{w^{*}}^{2}[0, T]} \leqslant C_{0} N^{-k}\left\|f\left(\frac{T}{2}(x+1)\right)\right\|_{H_{w}^{k}(-1,1)^{\prime}}
$$

where $\mathrm{C}_{0}$ is a positive constant, which depends on selected norm and is independent of $\mathrm{f}\left(\frac{\mathrm{T}}{2}(\mathrm{x}+1)\right)$ and $\mathrm{N}$.

Proof. By using change of the variable $t=2\left(\frac{x}{T}\right)-1$, we obtain

$$
\begin{aligned}
\left\|f(x)-f_{N}^{*}(x)\right\|_{L_{w^{*}}^{2}[0, T]}^{2} & =\int_{0}^{T}\left|f(x)-f_{N}^{*}(x)\right|^{2} w^{*}(x) d x \\
& =\int_{-1}^{1}\left|f\left(\frac{T}{2}(t+1)\right)-f_{N}^{*}\left(\frac{T}{2}(t+1)\right)\right|^{2} w(t) d t \\
& =\left\|f\left(\frac{T}{2}(t+1)\right)-f_{N}^{*}\left(\frac{T}{2}(t+1)\right)\right\|_{L_{w}^{2}[-1,1]}^{2}
\end{aligned}
$$

So, from Theorem 2.1, we have

$$
\left\|f(x)-f_{N}^{*}(x)\right\|_{L_{w^{*}}^{2}[0, T]} \leqslant C_{0} N^{-k}\left\|f\left(\frac{T}{2}(x+1)\right)\right\|_{H_{w}^{k}(-1,1)} .
$$

\subsection{Modified generalized Laguerre functions}

Here, we introduce the orthogonal system of modified generalized Laguerre functions, which are derived from generalized Laguerre polynomials $[9,20,39,41]$. We remind that in spite of orthogonality of Laguerre polynomials on $[0, \infty)$, these basis functions practically are not efficient. Some modifications and adjustments are necessary to enhance the competence of these approximation tools and overcome the difficulty of heavy computations.

\subsubsection{Generalized Laguerre polynomials}

The well-known generalized Laguerre polynomials (GLPs), denoted by $\mathcal{L}_{\mathfrak{n}}^{\alpha}(x)$, are orthogonal on the half line $[0,+\infty)$ with respect to the weight function $w_{\alpha}(x)=x^{\alpha} e^{-x}$, where $\alpha>-1$ [41]. The orthogonal- 
ity relation is written as

$$
\int_{0}^{+\infty} \mathcal{L}_{\mathrm{n}}^{\alpha}(x) \mathcal{L}_{\mathrm{m}}^{\alpha}(x) w_{\alpha}(x) \mathrm{d} x=\frac{\Gamma(\mathrm{n}+\alpha+1)}{\mathrm{n} !} \delta_{\mathrm{n}, \mathrm{m}}
$$

$\mathcal{L}_{\mathfrak{n}}^{\alpha}(x)$ is the $n^{\text {th }}$ eigenfunction of the Sturm-Liouville problem

$$
x \frac{\mathrm{d}^{2}}{\mathrm{~d} x^{2}} \mathcal{L}_{\mathrm{n}}^{\alpha}(\mathrm{x})+(\alpha+1-x) \frac{\mathrm{d}}{\mathrm{d} x} \mathcal{L}_{\mathrm{n}}^{\alpha}(\mathrm{x})+\lambda_{\mathrm{n}} \mathcal{L}_{\mathrm{n}}^{\alpha}(\mathrm{x})=0, \quad \mathrm{n}=0,1, \ldots,
$$

with the corresponding eigenvalue $\lambda_{n}=n$ and the normalizing condition $\mathcal{L}_{n}^{\alpha}(0)=\left(\begin{array}{c}n+\alpha \\ \alpha\end{array}\right)$. The analytic form of the generalized Laguerre polynomials $\mathcal{L}_{n}^{\alpha}(x)$ of degree $n$ is given by

$$
\mathcal{L}_{n}^{\alpha}(x)=\sum_{k=0}^{n} \frac{(-1)^{k}}{k !}\left(\begin{array}{l}
n+\alpha \\
n-k
\end{array}\right) x^{k} .
$$

The generalized Laguerre polynomials are defined with the following recurrence formula

$$
n \mathcal{L}_{n}^{\alpha}(x)=(2 n-1+\alpha-x) \mathcal{L}_{n-1}^{\alpha}(x)-(n+\alpha-1) \mathcal{L}_{n-2}^{\alpha}(x), \quad n=1,2, \ldots,
$$

where

$$
\mathcal{L}_{0}^{\alpha}(x)=1, \quad \mathcal{L}_{1}^{\alpha}(x)=1+\alpha-x .
$$

Theorem 2.3 ([42]). Let $\alpha>-1, \mathrm{c}$ and $\omega$ are fixed positive constants, and $\mathrm{cn}^{-1} \leqslant x \leqslant \omega$. Then

$$
\mathcal{L}_{n}^{\alpha}(x)=\pi^{-\frac{1}{2}} e^{\frac{x}{2}} x^{-\frac{\alpha}{2}-\frac{1}{4}} n^{\frac{\alpha}{2}-\frac{1}{4}}\left[\cos \left(2(n x)^{\frac{1}{2}}-\frac{\alpha \pi}{2}-\frac{\pi}{4}\right)+(n x)^{-\frac{1}{2}} O(1)\right] .
$$

Theorem 2.3 shows that the GLPs have a rapid growth as $x$ approaches to infinity and as $n$ increases. Also, we have the following uniform upper bounds for GLPs [19]:

$$
\left|\mathcal{L}_{n}^{\alpha}(x)\right| \leqslant \begin{cases}\frac{\Gamma(n+\alpha+1)}{n ! \Gamma(\alpha+1)} e^{\frac{x}{2}}, & \alpha \geqslant 0, \\ {\left[2-\frac{\Gamma(n+\alpha+1)}{n ! \Gamma(\alpha+1)}\right] e^{\frac{x}{2}},} & -1<\alpha<0 .\end{cases}
$$

\subsubsection{Generalized and modified generalized Laguerre functions}

In many problems in unbounded domains, the exact solutions decay algebraically or exponentially at infinity. So, the generalized Laguerre polynomials are not very useful yet in practice for finding the approximate solutions of these problems. Therefore, we consider the generalized Laguerre functions (GLFs) defined by [41]

$$
\mathrm{GL}_{\mathrm{n}}^{\alpha}(x)=e^{-\frac{x}{2}} \mathcal{L}_{\mathfrak{n}}^{\alpha}(x), \quad \mathrm{n}=0,1,2, \ldots
$$

By (2.1), the GLFs are orthogonal with respect to the weight function $\tilde{w}_{\alpha}(x)=x^{\alpha}$, i.e.,

$$
\int_{0}^{+\infty} \mathrm{GL}_{n}^{\alpha}(x) \mathrm{GL}_{m}^{\alpha}(x) \tilde{w}_{\alpha}(x) \mathrm{d} x=\frac{\Gamma(\mathrm{n}+\alpha+1)}{n !} \delta_{n, m} .
$$

The modified generalized Laguerre functions (MGLFs), denoted by $\mathrm{ML}_{n}^{\alpha, \mathrm{L}}(\mathrm{x})$, are defined as follows [39]:

$$
M L_{n}^{\alpha, L}(x)=G_{n}^{\alpha}\left(\frac{x}{L}\right), \quad n=0,1,2, \ldots,
$$

where the parameter $L$ is a positive scaling factor that is called mapping parameter.

Remark 2.4. Boyd in $[12,15]$ offered guidelines for optimizing the map parameter L for rational Chebyshev functions, which is useful for the proposed method in this paper, too. For the map parameter $L$ we have the following results $[12,15,23]$. 
- In general, there is no way to avoid a small amount of trial and error in choosing $L$ when solving problems on an unbounded domain.

- The optimum value of $L$ varies with the number of collocation points.

- Few experimentations are usually sufficient to determine a suitable value of $L$ because near the optimum value of $L$, the accuracy is insensitive to the precise value of $L$.

- The parameter L is a scaling/stretching factor, which can be used for the fine tuning of the spacing of collocation points.

$M L_{n}^{\alpha, L}(x)$ is the $n^{\text {th }}$ eigenfunction of the singular Sturm-Liouville problem

$$
\mathrm{Lx}^{-\alpha} e^{\frac{x}{2 \mathrm{~L}}} \frac{\mathrm{d}}{\mathrm{d} x}\left[x^{\alpha+1} e^{-\frac{x}{2 \mathrm{~L}}} \frac{\mathrm{d}}{\mathrm{dx}} M \mathrm{~L}_{\mathrm{n}}^{\alpha, \mathrm{L}}(\mathrm{x})\right]+\mathrm{nM} \mathrm{L}_{\mathrm{n}}^{\alpha, \mathrm{L}}(\mathrm{x})=0, \quad \mathrm{n}=0,1,2, \ldots,
$$

and by equations (2.2), (2.4) and (2.5), it satisfies in the following recurrence relation

$$
n M L_{n}^{\alpha, L}(x)=\left(2 n-1+\alpha-\frac{x}{L}\right) M L_{n-1}^{\alpha, L}(x)-(n+\alpha-1) M L_{n-2}^{\alpha, L}(x), \quad n=1,2, \ldots,
$$

where

$$
\mathrm{ML}_{0}^{\alpha, \mathrm{L}}(\mathrm{x})=e^{-\frac{x}{2 \mathrm{~L}}}, \quad \mathrm{ML}_{1}^{\alpha, \mathrm{L}}(x)=\left(1+\alpha-\frac{x}{\mathrm{~L}}\right) e^{-\frac{x}{2 \mathrm{~L}}} .
$$

This formula allows a stable evaluation of the MGLFs. Indeed, in contrast to the GLPs (see Theorem 2.3), the MGLFs are well-behaved with the decay property

$$
\left|M L_{n}^{\alpha, L}(x)\right| \rightarrow 0, \quad \text { as } x \rightarrow+\infty .
$$

Let $w_{\alpha, \mathrm{L}}(\mathrm{x})=\left(\frac{\mathrm{x}}{\mathrm{L}}\right)^{\alpha}$ denotes a non-negative, integrable, real-valued function over the interval $\mathrm{I}=[0,+\infty)$. We define

$$
\mathrm{L}_{w_{\alpha, \mathrm{L}}}^{2}(\mathrm{I})=\left\{\mathrm{f}: \mathrm{I} \longrightarrow \mathbb{R} \mid \mathrm{f} \text { is measurable and }\|\mathrm{f}\|_{w_{\alpha, \mathrm{L}}}<\infty\right\},
$$

where

$$
\|f\|_{\mathcal{w}_{\alpha, \mathrm{L}}}=\left(\int_{0}^{+\infty}|\mathrm{f}(\mathrm{x})|^{2} w_{\alpha, \mathrm{L}}(\mathrm{x}) \mathrm{d} x\right)^{\frac{1}{2}},
$$

is the norm induced by the scalar product

$$
\langle\mathrm{f}, \mathrm{g}\rangle_{w_{\alpha, \mathrm{L}}}=\int_{0}^{+\infty} \mathrm{f}(\mathrm{x}) \mathrm{g}(\mathrm{x}) w_{\alpha, \mathrm{L}}(\mathrm{x}) \mathrm{d} x
$$

Thus, $\left\{\mathrm{ML}_{\mathfrak{n}}^{\alpha, \mathrm{L}}(x)\right\}_{\mathfrak{n}=0}^{+\infty}$ is an orthogonal system in $\mathrm{L}_{w_{\alpha, \mathrm{L}}}^{2}(\mathrm{I})$, i.e.,

$$
\int_{0}^{+\infty} \mathrm{ML}_{\mathrm{n}}^{\alpha, \mathrm{L}}(x) \mathrm{ML}_{\mathrm{m}}^{\alpha, \mathrm{L}}(x) w_{\alpha, \mathrm{L}}(x) \mathrm{d} x=\frac{\mathrm{L} \Gamma(\mathrm{n}+\alpha+1)}{\mathrm{n} !} \delta_{\mathrm{n}, \mathrm{m}} .
$$

A function $f(x) \in L_{w_{\alpha, L}}^{2}$ (I) can be approximated by modified generalized Laguerre functions as

$$
f(x)=\sum_{j=0}^{\infty} f_{j} M L_{j}^{\alpha, L}(x),
$$

where the coefficients $f_{j}$ are given by

$$
f_{j}=\frac{j !\left\langle f(x), M L_{j}^{\alpha, L}(x)\right\rangle_{w_{\alpha, L}}}{L \Gamma(j+\alpha+1)}, j=0,1,2, \ldots
$$

\section{Description of the method}

In this section, to solve problem $(1.1)$ on $[0, T] \times[0, A]$ with respect to the initial conditions given by equations (1.2) and (1.3), we first approximate $u(t, x)$ by the $M+1$ shifted Chebyshev polynomials and 
$\mathrm{N}+1$ modified generalized Laguerre functions as

$$
u(t, x) \simeq u_{M, N}(t, x)=\sum_{i=0}^{M} \sum_{j=0}^{N} u_{i j} T_{i}^{*}(t) M L_{j}^{\alpha, L}(x) .
$$

Also, to construct the mesh for the covering of the domain, we consider the following two distributions of nodes

$$
\begin{aligned}
& \text { Case I: } \begin{cases}t_{i}=\frac{i T}{M}, \quad i=0,1, \ldots, M, \\
x_{j}=\frac{j A}{N}, \quad j=0,1, \ldots, N,\end{cases} \\
& \text { Case II: } \begin{cases}t_{i}=\frac{T}{2}\left(\cos \left(\frac{(M-i) \pi}{M}\right)+1\right), & i=0,1, \ldots, M, \\
x_{j}=\text { zeros of } \mathcal{L}_{N+1}^{0}(x), & j=0,1, \ldots, N .\end{cases}
\end{aligned}
$$

The distribution of 169 nodal points with the domain $[0,1] \times[0,100](T=1, A=100, \alpha=0)$ is depicted in Figure 1. According to Legendre-Gauss quadrature rule, by substituting (3.1) in (1.4), $U(t)$ can be

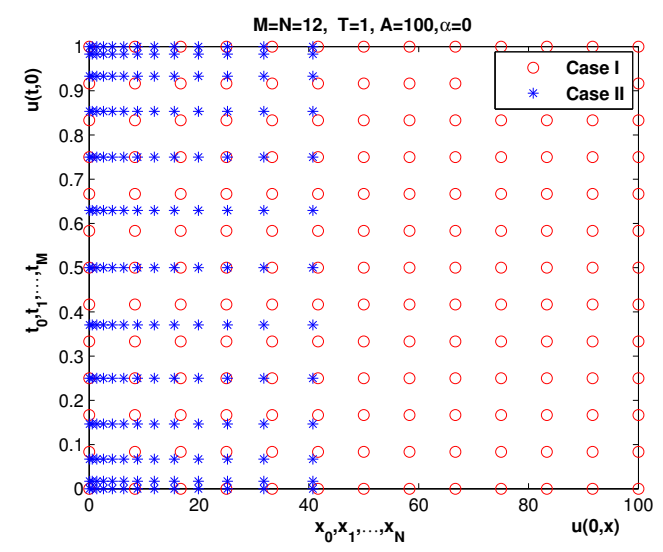

Figure 1: Distribution of nodes in cases I and II for $\mathrm{M}=\mathrm{N}=12$.

evaluated as follows

$$
\mathrm{u}(\mathrm{t}) \simeq \mathrm{u}_{M}(\mathrm{t})=\int_{0}^{A} \mathrm{u}_{M, N}(t, s) \mathrm{d} s \simeq \frac{A}{2} \sum_{j=0}^{p} w_{j} u_{M, N}\left(t, \frac{A}{2} \xi_{j}+\frac{A}{2}\right),
$$

where distinct nodes $\left\{\xi_{j}\right\}_{j=0}^{p}$ are the zeros of Legendre polynomial $L_{p+1}(t)$ and $\left\{w_{j}\right\}_{j=0}^{p}$ are corresponding weights and given in [16] as follows

$$
w_{j}=\frac{2}{\left(1-\xi_{j}^{2}\right)\left[L_{p+1}^{\prime}\left(\xi_{j}\right)\right]^{2}}, \quad j=0,1, \ldots, p .
$$

By substituting equations (3.1) and (3.2) in equation (1.1), we obtain the following residual function

$$
\operatorname{RES}(t, x)=\frac{\partial u_{M, N}(t, x)}{\partial t}+\frac{\partial u_{M, N}(t, x)}{\partial x}+\left[d_{1}(x)+d_{2}(x) u_{M}(t)\right] u_{M, N}(t, x) .
$$

By collocating equation (3.3) in $M N$ points $\left(t_{i}, x_{j}\right), i=0,1, \ldots, M-1, j=0,1, \ldots, N-1$, we obtain

$$
\operatorname{RES}\left(t_{i}, x_{j}\right)=0, \quad i=0,1, \ldots, M-1 \quad j=0,1, \ldots, N-1 .
$$

Replacing equations (3.1) and (3.2) into the initial conditions (1.2) and (1.3) we get

$$
\begin{aligned}
& u_{M, N}(0, x)=u_{0}(x), \\
& u_{M, N}(t, 0)=\int_{a}^{A}\left[b_{1}(s)-b_{2}(s) u_{M}(t)\right] u_{M, N}(t, s) d s .
\end{aligned}
$$

By using Legendre-Gauss quadrature rule, we can approximate the integral in the right hand of (3.6) as 
follows:

$$
u_{M, N}(t, 0)=\frac{A-a}{2} \sum_{j=0}^{p} w_{j}\left[b_{1}\left(\frac{A-a}{2}\left(\xi_{j}+1\right)\right)-b_{2}\left(\frac{A-a}{2}\left(\xi_{j}+1\right)\right) u_{M}(t)\right] u_{M, N}\left(t, \frac{A-a}{2}\left(\xi_{j}+1\right)\right) .
$$

Collocating equations (3.5) and (3.7) in $N+1$ points $x_{j}, j=0,1, \ldots, N$ and $M$ points $t_{i}, i=1,2, \ldots, M$, respectively, we have

$$
\begin{aligned}
& u_{M, N}\left(0, x_{j}\right)-u_{0}\left(x_{j}\right)=0, \quad j=0,1, \ldots, N \\
& u_{M, N}\left(t_{j}, 0\right)-\frac{A-a}{2} \sum_{j=0}^{p} w_{j}\left[b_{1}\left(\frac{A-a}{2}\left(\xi_{j}+1\right)\right)\right. \\
& \left.\quad-b_{2}\left(\frac{A-a}{2}\left(\xi_{j}+1\right)\right) u_{M}\left(t_{j}\right)\right] u_{M, N}\left(t_{j}, \frac{A-a}{2}\left(\xi_{j}+1\right)\right)=0, \quad i=1,2, \ldots, M .
\end{aligned}
$$

Equation (3.4) together with equations (3.8) and (3.9) give a $(M+1) \times(N+1)$ system of nonlinear equations, which can be solved for $u_{i j}$ 's, using Newton's iterative method. So the unknown function $u_{M, N}(t, x)$ can be calculated.

\section{Numerical results}

In this section some numerical examples are illustrated to demonstrate the efficiency and applicability of the proposed method. All computations are run using Maple 17 and MATLAB R2015a softwares on a Core(TM) i7 PC Laptop with $2.10 \mathrm{GHz}$ of CPU and $6 \mathrm{~GB}$ of RAM. In all examples, we set parameters $\alpha=0, p=40$, and $A=100$. Also, all the examples are solved with two distributions of nodes I and II in domain of the problem. For estimating the error of the numerical solution we use the error function $e_{M, N}(t, x)$ defined by

$$
e_{M, N}(t, x)=u(t, x)-u_{M, N}(t, x)
$$

Here, we have calculated the computational orders of the MGLC method with the following formula

$$
\text { C-order }=\frac{\log \left(\frac{\left\|e_{M_{2}, N}(t, x)\right\|_{\infty}}{\left\|e_{M_{1}, N}(t, x)\right\|_{\infty}}\right)}{\log \left(\frac{M_{1}}{M_{2}}\right)},
$$

where $\left\|e_{M_{1}, N}(t, x)\right\|_{\infty}$ and $\left\|e_{M_{2}, N}(t, x)\right\|_{\infty}$ are maximum absolute errors corresponding to grids with mesh size $M_{1}$ and $M_{2}$, respectively, for fixed value of $N$. This index obviously shows the exponential rate of convergence. Precisely, a larger number of basis function intensively impacts on decreasing absolute error values. In all examples, we used the following methods for finding a suitable map parameter L [12-15].

First method: We solve the problem and look at the spectral coefficients, plotted as absolute values on a log-linear plot, for several different values of L. Obviously, if the magnitudes of the coefficients are rapidly decreasing, the spectral series is accurate $[12,15]$.

Second method: We depict the maximum absolute residual error versus $L$ for a fixed values of $M$ and $N$. This curve has a "V-shape" for reasons explained in more detail in $[12,13,15]$.

Example 4.1. Consider the following nonlinear age-structured population model [17, 32, 35, 45]

$$
\begin{aligned}
\frac{\partial u(t, x)}{\partial t}+\frac{\partial u(t, x)}{\partial x} & =-u(t) u(t, x), \quad 0 \leqslant t, \quad 0 \leqslant x<A, \\
u(0, x) & =\frac{e^{-x}}{2}, \quad 0 \leqslant x<A
\end{aligned}
$$




$$
\begin{aligned}
u(t, 0) & =u(t), \quad 0 \leqslant t, \\
u(t) & =\int_{0}^{A} u(t, s) d s, \quad 0 \leqslant t,
\end{aligned}
$$

which $A=+\infty$ and has the exact solution

$$
u(t, x)=\frac{e^{-x}}{e^{-t}+1}, \quad x \geqslant 0, \quad t \geqslant 0 .
$$

Table 1 shows the maximum absolute values of errors and computational orders for $T=1, N=2$, $\mathrm{L}=0.5$ and different values of $M$, using the method proposed in Section 3. From table, we can see that as the number of nodes increases, the accuracy improves. Also, the distribution of nodes in case II creates more accurate results. However, the accuracy of the distribution of nodes in case I is acceptable. In Table 2, we compare the absolute errors obtained for Example 4.1 using the MGLC method with $N=2, M=$ $5, \mathrm{~L}=0.5$ and the operational matrices method based on Bernstein polynomials presented in [45]. The comparison of the absolute error between HAM and OHAM with four terms and the MGLC method with $N=2, M=12, L=0.5$ are given in Table 3 for various values of $(t, x) \in[0,1] \times[0,50]$. Figure 3 shows the logarithmic of absolute values $u_{i j}$ as computed for case II with $T=1, N=3, M=12$ and several scaling factor $L$. We observe that the optimum value of parameter $L$ is about $L=0.5$. Figure 2 , shows the absolute error function $\left|e_{M, N}(t, x)\right|$ obtained by the propose method with $T=5([0,5] \times[0,100]), N=2, L=0.5$ and $M=10$. According to Figure 2, we find that the presented method provides accurate results in large domain even for small N. Also, Figure 12 (a) shows the graphs of maximum absolute residual errors in approximating $u(t, x)$ for case II with $T=1, N=2$ and various values of L. We can see the optimum value of parameter $L$ is about $L=0.5$, similar to Figure 3 .

Table 1: Errors and computational orders obtained for Example 4.1 with $\mathrm{T}=1, \mathrm{~N}=2$, and $\mathrm{L}=0.5$.

\begin{tabular}{llllll}
\hline \multirow{2}{*}{$\mathrm{N}$} & Case I & & & Case II & \\
\cline { 2 - 3 } \cline { 5 - 6 } & $\left\|e_{M, N}\right\|_{\infty}$ & C-order & & $\left\|e_{M, N}\right\|_{\infty}$ & C-order \\
\hline 4 & $4.2086 \times 10^{-5}$ & - & & $1.2166 \times 10^{-5}$ & - \\
6 & $3.1568 \times 10^{-7}$ & 12.0669 & & $3.5128 \times 10^{-8}$ & 14.4215 \\
8 & $1.6330 \times 10^{-10}$ & 26.3029 & & $6.0148 \times 10^{-11}$ & 22.1423 \\
10 & $5.6723 \times 10^{-11}$ & 4.7387 & & $1.0794 \times 10^{-12}$ & 18.0171 \\
12 & $1.5204 \times 10^{-12}$ & 19.8506 & & $1.2636 \times 10^{-14}$ & 24.3943 \\
14 & $2.8998 \times 10^{-14}$ & 25.6859 & & $1.0584 \times 10^{-16}$ & 31.0240 \\
\hline
\end{tabular}

Table 2: Absolute error obtained for Example 4.1 on $[0,1] \times[0,20]$ and $[0,1] \times[0,100]$ when $L=0.5$.

\begin{tabular}{llllll}
\hline$(\mathrm{t}, \mathrm{x})$ & $\begin{array}{l}\text { Yousefi et al. [45] } \\
(\mathrm{m}=5, \mathrm{n}=10)\end{array}$ & $\begin{array}{l}\text { MGLC (Case I) } \\
(\mathrm{N}=2, \mathrm{M}=5)\end{array}$ & $(\mathrm{t}, \mathrm{x})$ & $\begin{array}{l}\text { Yousefi et al. [45] } \\
(\mathrm{m}=5, \mathrm{n}=10)\end{array}$ & $\begin{array}{l}\text { MGLC (Case I) } \\
(\mathrm{N}=2, \mathrm{M}=5)\end{array}$ \\
\hline$(0,0)$ & $1.71 \times 10^{-3}$ & 0.00 & $(0,0)$ & $1.49 \times 10^{-1}$ & 0.00 \\
$(0.1,2)$ & $3.74 \times 10^{-3}$ & $3.06 \times 10^{-8}$ & $(0.1,10)$ & $4.70 \times 10^{-3}$ & $1.02 \times 10^{-11}$ \\
$(0.2,4)$ & $6.74 \times 10^{-4}$ & $6.67 \times 10^{-9}$ & $(0.2,20)$ & $1.98 \times 10^{-3}$ & $7.44 \times 10^{-16}$ \\
$(0.3,6)$ & $4.58 \times 10^{-4}$ & $6.84 \times 10^{-10}$ & $(0.3,30)$ & $5.08 \times 10^{-3}$ & $2.55 \times 10^{-20}$ \\
$(0.4,8)$ & $2.08 \times 10^{-4}$ & $6.60 \times 10^{-11}$ & $(0.4,40)$ & $6.28 \times 10^{-3}$ & $8.24 \times 10^{-25}$ \\
$(0.5,10)$ & $8.91 \times 10^{-5}$ & $1.21 \times 10^{-11}$ & $(0.5,50)$ & $3.56 \times 10^{-3}$ & $5.14 \times 10^{-29}$ \\
$(0.6,12)$ & $1.08 \times 10^{-4}$ & $2.13 \times 10^{-12}$ & $(0.6,60)$ & $1.08 \times 10^{-3}$ & $3.06 \times 10^{-33}$ \\
$(0.7,14)$ & $2.10 \times 10^{-4}$ & $1.43 \times 10^{-13}$ & $(0.7,70)$ & $4.19 \times 10^{-3}$ & $7.07 \times 10^{-42}$ \\
$(0.8,16)$ & $2.20 \times 10^{-4}$ & $1.22 \times 10^{-14}$ & $(0.8,80)$ & $4.90 \times 10^{-3}$ & $1.75 \times 10^{-46}$ \\
$(0.9,18)$ & $2.45 \times 10^{-4}$ & $1.55 \times 10^{-14}$ & $(0.9,90)$ & $5.33 \times 10^{-3}$ & $8.50 \times 10^{-46}$ \\
$(1,20)$ & $7.73 \times 10^{-4}$ & $1.60 \times 10^{-14}$ & $(1,100)$ & $1.63 \times 10^{-2}$ & $2.90 \times 10^{-49}$ \\
\hline
\end{tabular}


Table 3: Comparison between absolute errors of HAM, OHAM and the MGLC method for Example 4.1 with $\mathrm{N}=2, \mathrm{M}=12$, and $\mathrm{L}=0.5$ on $[0,1] \times[0,50]$.

\begin{tabular}{|c|c|c|c|c|c|c|}
\hline \multirow[t]{2}{*}{$x$} & \multicolumn{3}{|l|}{$t=0.1$} & \multicolumn{3}{|l|}{$t=1$} \\
\hline & HAM [21] & OHAM [21] & MGLC (Case II) & HAM [21] & OHAM [21] & MGLC (Case II) \\
\hline 0 & $2.08 \times 10^{-8}$ & $7.54 \times 10^{-5}$ & $8.02 \times 10^{-17}$ & $1.89 \times 10^{-3}$ & $3.30 \times 10^{-6}$ & $1.26 \times 10^{-14}$ \\
\hline 5 & $1.40 \times 10^{-10}$ & $5.08 \times 10^{-5}$ & $5.41 \times 10^{-19}$ & $1.27 \times 10^{-5}$ & $2.22 \times 10^{-6}$ & $8.51 \times 10^{-17}$ \\
\hline 10 & $9.45 \times 10^{-13}$ & $3.42 \times 10^{-9}$ & $3.64 \times 10^{-21}$ & $8.59 \times 10^{-8}$ & $1.50 \times 10^{-10}$ & $5.74 \times 10^{-19}$ \\
\hline 20 & $4.29 \times 10^{-17}$ & $1.55 \times 10^{-13}$ & $1.65 \times 10^{-25}$ & $3.90 \times 10^{-12}$ & $6.80 \times 10^{-15}$ & $2.60 \times 10^{-23}$ \\
\hline 30 & $1.95 \times 10^{-21}$ & $7.05 \times 10^{-18}$ & $7.51 \times 10^{-30}$ & $1.77 \times 10^{-16}$ & $3.09 \times 10^{-19}$ & $1.18 \times 10^{-27}$ \\
\hline 40 & $8.84 \times 10^{-26}$ & $3.20 \times 10^{-22}$ & $3.41 \times 10^{-34}$ & $8.04 \times 10^{-21}$ & $1.40 \times 10^{-23}$ & $5.37 \times 10^{-32}$ \\
\hline 50 & $4.01 \times 10^{-30}$ & $1.45 \times 10^{-26}$ & $1.55 \times 10^{-38}$ & $3.65 \times 10^{-25}$ & $6.36 \times 10^{-28}$ & $2.44 \times 10^{-36}$ \\
\hline
\end{tabular}

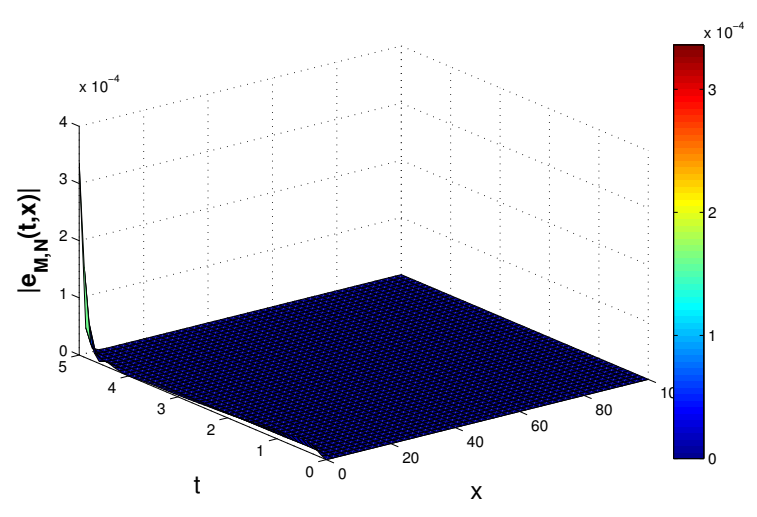

(a)

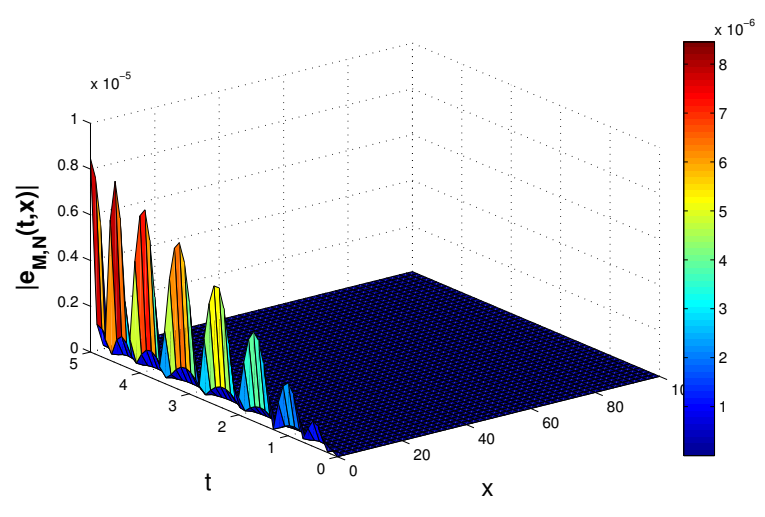

(b)

Figure 2: Absolute error functions $\left|e_{M, N}(t, x)\right|$, for Case I (a) and Case II (b), where $M=10, N=2, T=5$, and $L=0.5$. See Example 4.1.

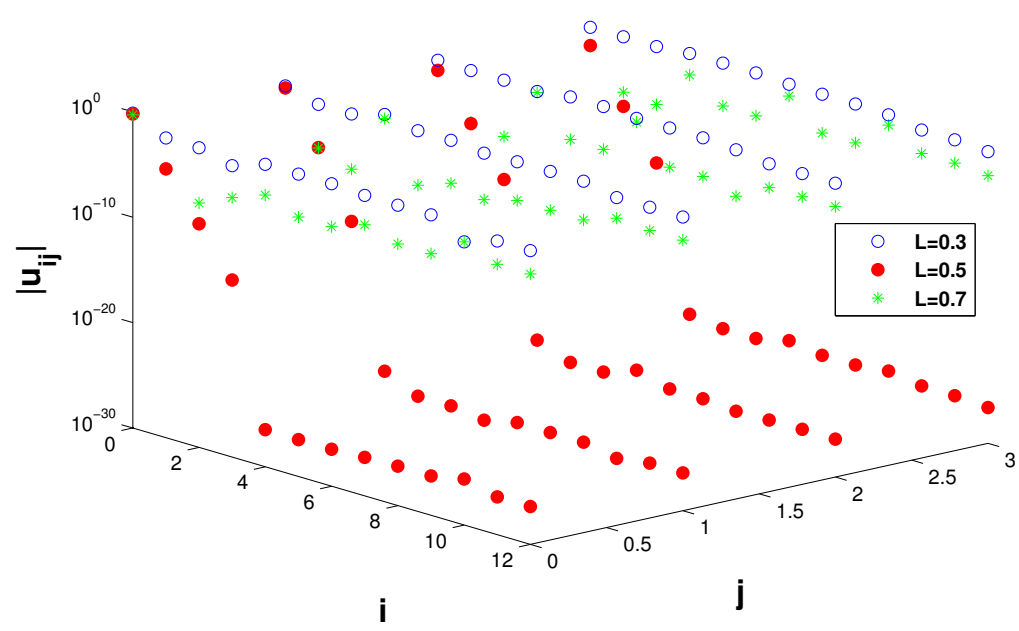

Figure 3: The logarithmic of absolute values of $u_{i j}$ versus $i$ and $j$ with $T=1, N=3, M=12$ and $L=0.3,0.5,0.7$ for Case II, Example 4.1.

Example 4.2. Consider the following nonlinear age-structured population model [32, 35, 45]

$$
\begin{aligned}
\frac{\partial u(t, x)}{\partial t}+\frac{\partial u(t, x)}{\partial x} & =-(1+u(t)) u(t, x), \quad 0 \leqslant t, \quad 0 \leqslant x<A, \\
u(0, x) & =\frac{e^{-x}}{2}, \quad 0 \leqslant x<A,
\end{aligned}
$$




$$
\begin{aligned}
u(t, 0) & =u(t), \quad 0 \leqslant t, \\
u(t) & =\int_{0}^{A} u(t, s) d s, \quad 0 \leqslant t,
\end{aligned}
$$

which $A=+\infty$ and has the exact solution

$$
u(t, x)=\frac{e^{-x}}{2+t^{\prime}}, \quad x \geqslant 0, \quad t \geqslant 0 .
$$

Numerical results are presented in Table 4 for $T=1, N=2, L=0.5$ and different values of $M$. In

Table 4: Errors and computational orders obtained for Example 4.2 with $T=1, \mathrm{~N}=2$ and $\mathrm{L}=0.5$.

\begin{tabular}{llllll}
\hline \multirow{2}{*}{$\mathrm{n}$} & \multicolumn{2}{l}{ Case I } & & & \multicolumn{2}{l}{ Case II } & \\
\cline { 2 - 3 } \cline { 5 - 6 } & $\left\|e_{M, N}\right\|_{\infty}$ & C-order & & $\left\|e_{M, N}\right\|_{\infty}$ & C-order \\
\hline 4 & $1.7703 \times 10^{-4}$ & - & & $5.1488 \times 10^{-5}$ & - \\
6 & $4.0011 \times 10^{-6}$ & 9.3467 & & $4.5664 \times 10^{-7}$ & 11.6538 \\
8 & $9.1175 \times 10^{-9}$ & 21.1488 & & $4.3599 \times 10^{-9}$ & 16.1687 \\
10 & $2.0763 \times 10^{-9}$ & 6.6307 & & $4.2796 \times 10^{-11}$ & 20.7210 \\
12 & $4.7168 \times 10^{-11}$ & 20.7580 & & $4.2573 \times 10^{-13}$ & 25.2872 \\
14 & $1.0687 \times 10^{-12}$ & 24.5686 & & $4.2671 \times 10^{-15}$ & 29.8596 \\
\hline
\end{tabular}

Table 5 we compare the absolute errors obtained using the MGLC method with $N=2, M=8, L=0.5$ and the method presented in [45].

Table 5: Absolute error obtained for Example 4.2 on $[0,1] \times[0,20]$ and $[0,1] \times[0,100]$ when $L=0.5$.

\begin{tabular}{llllll}
\hline$(\mathrm{t}, \mathrm{x})$ & $\begin{array}{l}\text { Yousefi et al. [45] } \\
(\mathrm{m}=5, \mathrm{n}=10)\end{array}$ & $\begin{array}{l}\text { MGLC (Case I) } \\
(\mathrm{N}=2, \mathrm{M}=5)\end{array}$ & $(\mathrm{t}, \mathrm{x})$ & $\begin{array}{l}\text { Yousefi et al. [45] } \\
(\mathrm{m}=5, \mathrm{n}=10)\end{array}$ & $\begin{array}{l}\text { MGLC (Case I) } \\
(\mathrm{N}=2, \mathrm{M}=5)\end{array}$ \\
\hline$(0,0)$ & $1.71 \times 10^{-3}$ & 0.00 & $(0,0)$ & $1.49 \times 10^{-1}$ & 0.00 \\
$(0.1,2)$ & $2.86 \times 10^{-3}$ & $2.28 \times 10^{-10}$ & $(0.1,10)$ & $4.70 \times 10^{-3}$ & $1.77 \times 10^{-13}$ \\
$(0.2,4)$ & $1.07 \times 10^{-3}$ & $7.06 \times 10^{-12}$ & $(0.2,20)$ & $1.98 \times 10^{-3}$ & $6.39 \times 10^{-18}$ \\
$(0.3,6)$ & $1.12 \times 10^{-4}$ & $4.49 \times 10^{-13}$ & $(0.3,30)$ & $5.08 \times 10^{-3}$ & $2.59 \times 10^{-22}$ \\
$(0.4,8)$ & $2.69 \times 10^{-4}$ & $3.03 \times 10^{-13}$ & $(0.4,40)$ & $6.28 \times 10^{-3}$ & $1.16 \times 10^{-26}$ \\
$(0.5,10)$ & $7.90 \times 10^{-5}$ & $8.15 \times 10^{-14}$ & $(0.5,50)$ & $3.56 \times 10^{-3}$ & $4.40 \times 10^{-31}$ \\
$(0.6,12)$ & $1.07 \times 10^{-4}$ & $2.27 \times 10^{-14}$ & $(0.6,60)$ & $1.08 \times 10^{-3}$ & $2.10 \times 10^{-35}$ \\
$(0.7,14)$ & $2.10 \times 10^{-4}$ & $4.41 \times 10^{-15}$ & $(0.7,70)$ & $4.19 \times 10^{-3}$ & $7.39 \times 10^{-40}$ \\
$(0.8,16)$ & $2.20 \times 10^{-4}$ & $8.86 \times 10^{-16}$ & $(0.8,80)$ & $4.90 \times 10^{-3}$ & $3.77 \times 10^{-44}$ \\
$(0.9,18)$ & $2.45 \times 10^{-4}$ & $1.75 \times 10^{-16}$ & $(0.9,90)$ & $5.33 \times 10^{-3}$ & $2.64 \times 10^{-48}$ \\
$(1,20)$ & $7.73 \times 10^{-4}$ & $1.66 \times 10^{-16}$ & $(1,100)$ & $1.63 \times 10^{-2}$ & $3.39 \times 10^{-51}$ \\
\hline
\end{tabular}

The comparison of the absolute error between HAM and OHAM with four terms and the MGLC method with $N=4, M=12, L=0.5$ are given in Table 6 for various values of $(t, x) \in[0,1] \times[0,50]$.

\begin{tabular}{|c|c|c|c|c|c|c|}
\hline \multirow[t]{2}{*}{$x$} & \multicolumn{3}{|l|}{$t=0.1$} & \multicolumn{3}{|l|}{$t=1$} \\
\hline & HAM [21] & OHAM [21] & MGLC (Case II) & HAM [21] & OHAM [21] & MGLC (Case II) \\
\hline 0 & $2.98 \times 10^{-6}$ & $1.18 \times 10^{-4}$ & $5.92 \times 10^{-15}$ & $2.08 \times 10^{-2}$ & $1.03 \times 10^{-6}$ & $4.26 \times 10^{-13}$ \\
\hline 5 & $2.01 \times 10^{-8}$ & $7.97 \times 10^{-7}$ & $3.99 \times 10^{-17}$ & $1.40 \times 10^{-4}$ & $6.94 \times 10^{-9}$ & $2.87 \times 10^{-15}$ \\
\hline 10 & $1.35 \times 10^{-10}$ & $5.37 \times 10^{-9}$ & $2.69 \times 10^{-19}$ & $9.46 \times 10^{-7}$ & $4.68 \times 10^{-11}$ & $1.93 \times 10^{-17}$ \\
\hline 20 & $6.13 \times 10^{-15}$ & $2.44 \times 10^{-13}$ & $1.22 \times 10^{-23}$ & $4.29 \times 10^{-11}$ & $2.12 \times 10^{-15}$ & $8.78 \times 10^{-22}$ \\
\hline 30 & $2.79 \times 10^{-19}$ & $1.11 \times 10^{-17}$ & $5.54 \times 10^{-28}$ & $1.95 \times 10^{-15}$ & $9.64 \times 10^{-20}$ & $3.98 \times 10^{-26}$ \\
\hline 40 & $1.26 \times 10^{-23}$ & $5.03 \times 10^{-22}$ & $2.52 \times 10^{-32}$ & $8.85 \times 10^{-20}$ & $4.38 \times 10^{-24}$ & $1.81 \times 10^{-30}$ \\
\hline 50 & $5.74 \times 10^{-28}$ & $2.28 \times 10^{-26}$ & $1.15 \times 10^{-36}$ & $4.02 \times 10^{-24}$ & $1.99 \times 10^{-28}$ & $8.21 \times 10^{-35}$ \\
\hline
\end{tabular}

Table 6: Comparison between absolute errors of HAM, OHAM and the MGLC method for Example 4.2 with $\mathrm{N}=4, \mathrm{M}=12$ and $\mathrm{L}=0.5$ on $[0,1] \times[0,50]$. 
The absolute error function $\left|e_{M, N}(t, x)\right|$ with $T=10([0,10] \times[0,100]), N=2, L=0.5$ and $M=16$ is shown in Figure 4. From Table 4 and Figure 4, we can see that the MGLC method provides accurate results.

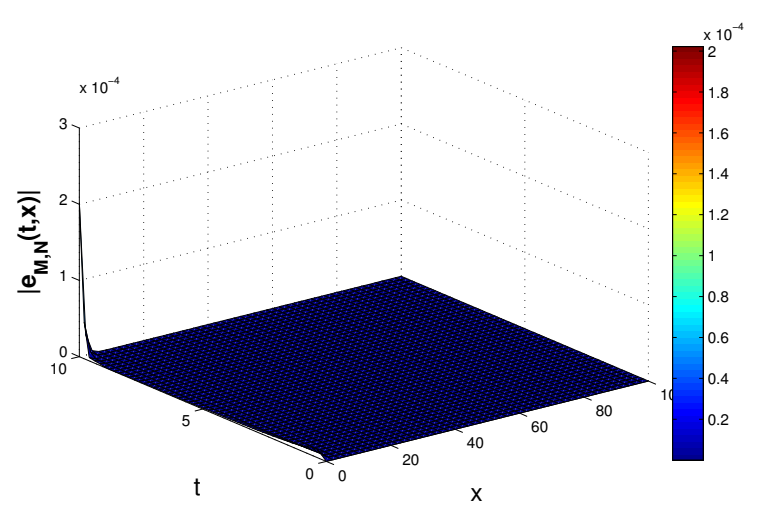

(a)

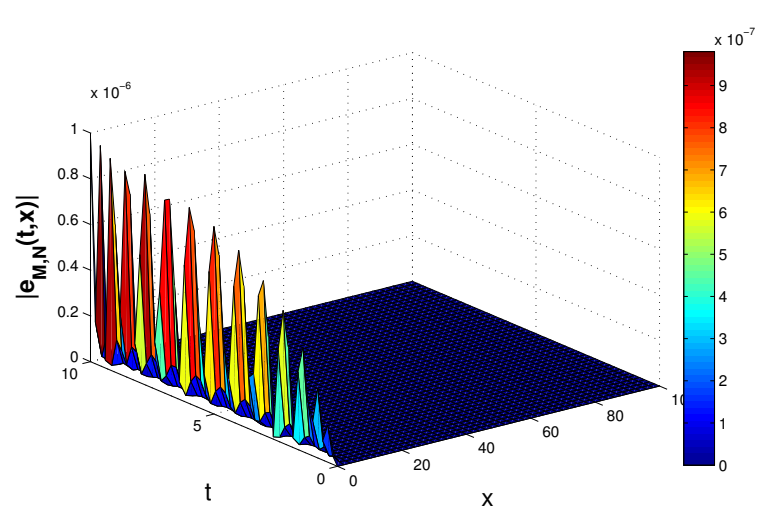

(b)

Figure 4: The absolute error function $\left|e_{M, N}(t, x)\right|$, for Case I (a) and Case II (b) where $M=16, N=2, T=10$, and $L=0.5$. See Example 4.2.

Figures 5 and 12 (b) show the logarithmic of absolute values $u_{i j}$ as computed for case II with $\mathrm{T}=$ $1, N=4, M=12$ and the maximum absolute residual errors for case II with $T=1, N=2$ and various values of $L$, respectively. In this example, we observe that the optimum value of parameter $L$ is about $\mathrm{L}=0.5$.

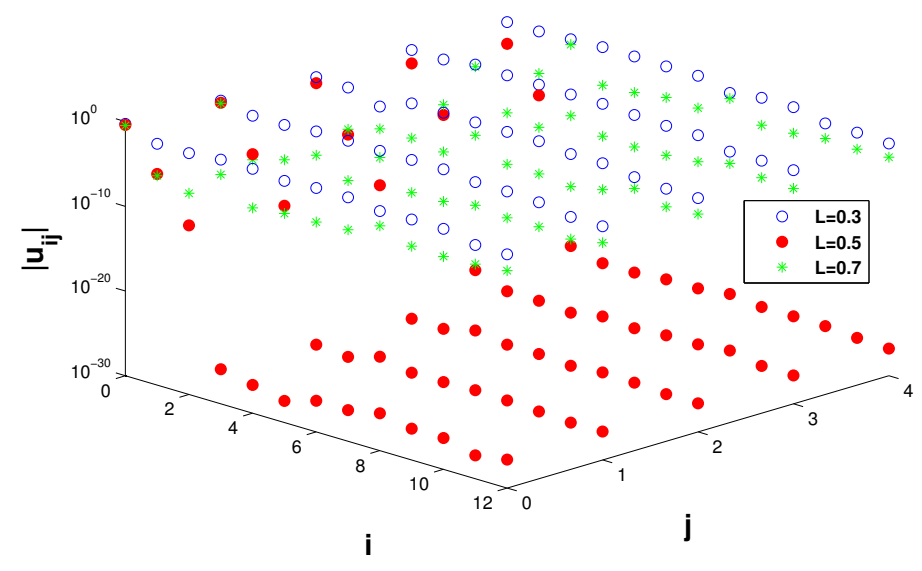

Figure 5: The logarithmic of absolute values of $u_{i j}$ versus $i$ and $j$ with $T=1, N=4, M=12$, and $L=0.3,0.5,0.7$ for Case II, Example 4.2.

Example 4.3. Consider the following nonlinear age-structured population model [5]

$$
\begin{aligned}
\frac{\partial u(t, x)}{\partial t}+\frac{\partial u(t, x)}{\partial x} & =-\left(2+\frac{4}{e} u(t)\right) u(t, x), \quad 0 \leqslant t, \quad 0 \leqslant x<A, \\
u(0, x) & =\frac{e^{1-2 x}}{4}, \quad 0 \leqslant x<A, \\
u(t, 0) & =2 u(t), \quad 0 \leqslant t, \\
u(t) & =\int_{0}^{A} u(t, s) d s, \quad 0 \leqslant t,
\end{aligned}
$$

which $A=+\infty$ and has the exact solution 


$$
u(t, x)=\frac{e^{1-2 x}}{4+2 t}, \quad x \geqslant 0, \quad t \geqslant 0
$$

We approximate $u(t, x)$ on $[0,1] \times[0,100]$ and present the the maximum absolute values of errors and computational orders for $\mathrm{N}=3, \mathrm{~L}=0.25$ and different values of $M$ in Table 7 .

Table 7: Errors and computational orders obtained for Example 4.3 with $\mathrm{T}=1, \mathrm{~N}=3$ and $\mathrm{L}=0.25$.

\begin{tabular}{|c|c|c|c|c|}
\hline \multirow[t]{2}{*}{$M$} & \multicolumn{2}{|l|}{ Case I } & \multicolumn{2}{|l|}{ Case II } \\
\hline & $\left\|e_{M, N}\right\|_{\infty}$ & C-order & $\left\|e_{M, N}\right\|_{\infty}$ & C-order \\
\hline 4 & $2.4061 \times 10^{-4}$ & - & $6.9979 \times 10^{-5}$ & - \\
\hline 6 & $5.4381 \times 10^{-6}$ & 9.3467 & $6.2064 \times 10^{-7}$ & 11.6538 \\
\hline 8 & $1.2391 \times 10^{-7}$ & 13.1452 & $5.9256 \times 10^{-9}$ & 16.1687 \\
\hline 10 & $2.8219 \times 10^{-9}$ & 16.9494 & $5.8055 \times 10^{-11}$ & 20.7295 \\
\hline 12 & $6.4021 \times 10^{-11}$ & 20.7653 & $6.6820 \times 10^{-13}$ & 24.4873 \\
\hline 14 & $1.3679 \times 10^{-12}$ & 24.9492 & $1.2458 \times 10^{-13}$ & 10.8961 \\
\hline
\end{tabular}

In Table 8, we compare the absolute errors obtained using the MGLC method with $\mathrm{N}=3, \mathrm{M}=8, \mathrm{~L}=$ 0.25 , and the method of [45].

Table 8: Absolute error obtained for Example 4.3 on $[0,1] \times[0,20]$ and $[0,1] \times[0,100]$ when $L=0.25$.

\begin{tabular}{llllll}
\hline$(\mathrm{t}, \mathrm{x})$ & $\begin{array}{l}\text { Yousefi et al. [45] } \\
(\mathrm{m}=5, \mathrm{n}=10)\end{array}$ & $\begin{array}{l}\text { MGLC (Case I) } \\
(\mathrm{N}=3, \mathrm{M}=8)\end{array}$ & $(\mathrm{t}, \mathrm{x})$ & $\begin{array}{l}\text { Yousefi et al. [45] } \\
(\mathrm{m}=5, \mathrm{n}=10)\end{array}$ & $\begin{array}{l}\text { MGLC (Case I) } \\
(\mathrm{N}=3, \mathrm{M}=8)\end{array}$ \\
\hline$(0,0)$ & $3.43 \times 10^{-2}$ & 0.00 & $(0,0)$ & $3.71 \times 10^{-1}$ & 0.00 \\
$(0.1,2)$ & $3.96 \times 10^{-3}$ & $9.72 \times 10^{-11}$ & $(0.1,10)$ & $2.28 \times 10^{-3}$ & $1.09 \times 10^{-17}$ \\
$(0.2,4)$ & $2.39 \times 10^{-3}$ & $1.41 \times 10^{-12}$ & $(0.2,20)$ & $1.07 \times 10^{-3}$ & $1.79 \times 10^{-26}$ \\
$(0.3,6)$ & $3.23 \times 10^{-3}$ & $2.31 \times 10^{-14}$ & $(0.3,30)$ & $5.74 \times 10^{-3}$ & $3.30 \times 10^{-35}$ \\
$(0.4,8)$ & $3.28 \times 10^{-3}$ & $4.17 \times 10^{-16}$ & $(0.4,40)$ & $7.83 \times 10^{-3}$ & $6.70 \times 10^{-44}$ \\
$(0.5,10)$ & $1.52 \times 10^{-3}$ & $6.39 \times 10^{-18}$ & $(0.5,50)$ & $4.76 \times 10^{-3}$ & $1.15 \times 10^{-52}$ \\
$(0.6,12)$ & $9.22 \times 10^{-4}$ & $1.23 \times 10^{-19}$ & $(0.6,60)$ & $1.06 \times 10^{-3}$ & $2.50 \times 10^{-61}$ \\
$(0.7,14)$ & $2.40 \times 10^{-3}$ & $1.75 \times 10^{-21}$ & $(0.7,70)$ & $5.12 \times 10^{-3}$ & $3.99 \times 10^{-70}$ \\
$(0.8,16)$ & $2.65 \times 10^{-3}$ & $3.59 \times 10^{-23}$ & $(0.8,80)$ & $6.14 \times 10^{-3}$ & $9.23 \times 10^{-79}$ \\
$(0.9,18)$ & $2.90 \times 10^{-3}$ & $1.01 \times 10^{-24}$ & $(0.9,90)$ & $6.68 \times 10^{-3}$ & $2.93 \times 10^{-87}$ \\
$(1,20)$ & $8.97 \times 10^{-3}$ & $5.26 \times 10^{-25}$ & $(1,100)$ & $2.04 \times 10^{-2}$ & $1.71 \times 10^{-94}$ \\
\hline
\end{tabular}

The absolute error function $\left|e_{M, N}(t, x)\right|$ with $T=10([0,10] \times[0,100]), N=3, L=0.25$ and $M=14$ is shown in Figure 6. This figure shows that the proposed method produces very good results on the large domain.

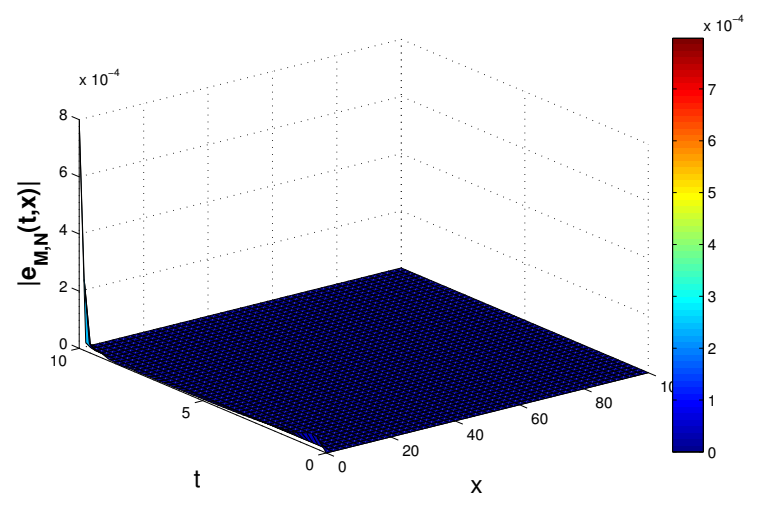

(a)

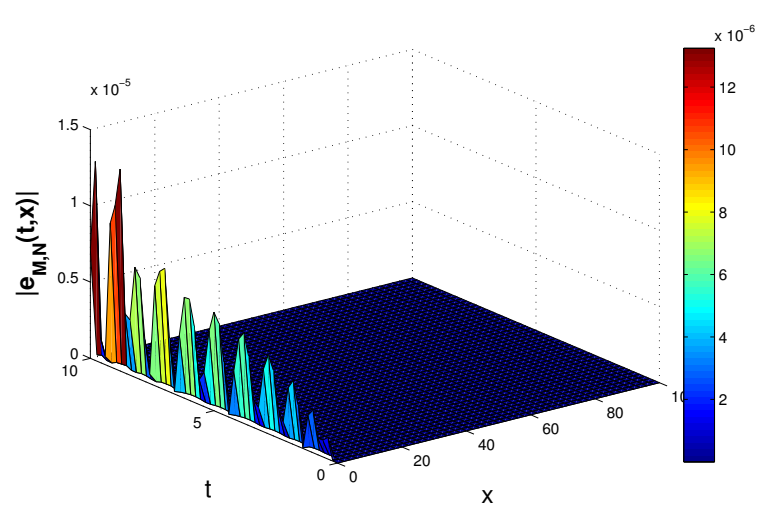

(b)

Figure 6: Absolute error function $\left|e_{M, N}(t, x)\right|$, for Case I (a) and Case II (b) where $M=14, N=3, T=10$ and $L=0.25$. See Example 4.3. 
Figures 7 and 12 (c) show the logarithmic of absolute values $u_{i j}$ as computed for case II with $\mathrm{T}=$ $1, N=4, M=12$ and the maximum absolute residual errors for case II with $T=1, N=3$ and various values of $L$, respectively. In this example, the optimum value of parameter $L$ is about $L=0.25$.

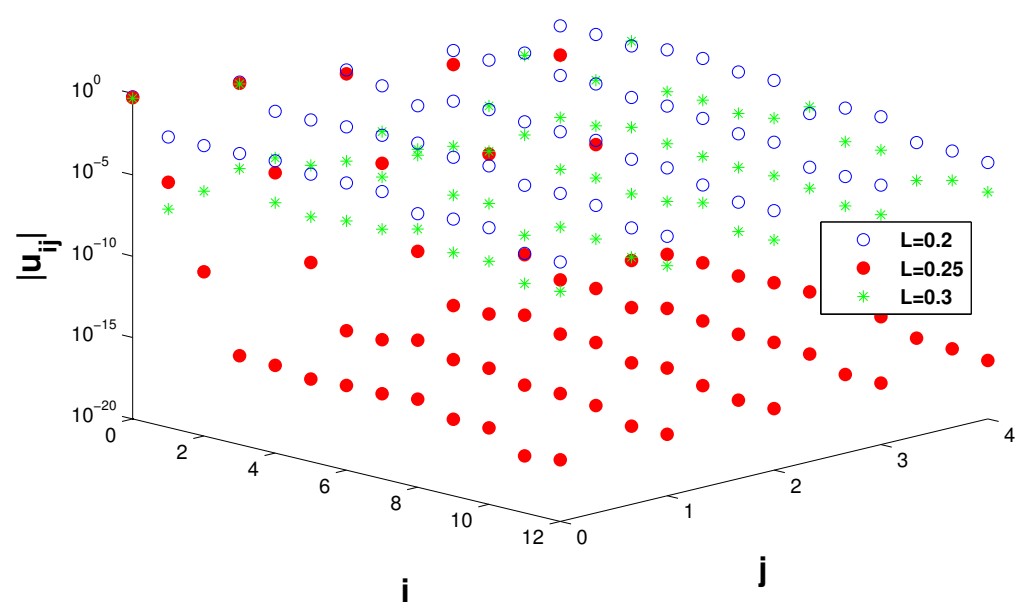

Figure 7: The logarithmic of absolute values of $u_{i j}$ versus $i$ and $j$ with $T=1, N=4, M=12$ and $L=0.2,0.25,0.3$ for Case II, Example 4.3.

Example 4.4. Consider the following nonlinear age-structured population model [5]

$$
\begin{aligned}
\frac{\partial u(t, x)}{\partial t}+\frac{\partial u(t, x)}{\partial x} & =-\left(\frac{1}{1-x}+u(t)\right) u(t, x), \quad 0 \leqslant t, \quad 0 \leqslant x<A, \\
u(0, x) & =4(1-x) e^{-\lambda x}, \quad 0 \leqslant x<A, \\
u(t, 0) & =4 u(t), \quad 0 \leqslant t, \\
u(t) & =\int_{0}^{A} u(t, s) d s, \quad 0 \leqslant t,
\end{aligned}
$$

which $\lambda=2$ and $A=+\infty$ and has the exact solution

$$
u(t, x)=4(1-x) e^{-\lambda x} \frac{\lambda}{1+(1-\lambda) e^{-\lambda t}}, \quad x \geqslant 0, \quad t \geqslant 0 .
$$

We approximate $u(t, x)$ on $[0,1] \times[0,100]$ and present the the maximum absolute values of errors and computational orders for $\mathrm{N}=2, \mathrm{~L}=0.25$ and different values of $\mathrm{M}$ in Table 9. In Table 10,

Table 9: Errors and computational orders obtained for Example 4.4 with $\mathrm{T}=1, \mathrm{~N}=2$ and $\mathrm{L}=0.25$.

\begin{tabular}{llllll}
\hline \multirow{2}{*}{$\mathrm{n}$} & Case I & & & Case II & \\
\cline { 2 - 3 } & $\left\|e_{M, N}\right\|_{\infty}$ & C-order & & $\left\|e_{M, N}\right\|_{\infty}$ & C-order \\
\hline 2 & $1.4546 \times 10^{-1}$ & - & & $1.4546 \times 10^{-1}$ & \\
4 & $2.3579 \times 10^{-3}$ & 5.9470 & & $7.3356 \times 10^{-4}$ & 7.6315 \\
6 & $5.0873 \times 10^{-4}$ & 3.7823 & & $5.5347 \times 10^{-5}$ & 6.3736 \\
8 & $3.5997 \times 10^{-5}$ & 9.2063 & & $1.6008 \times 10^{-6}$ & 12.3161 \\
10 & $1.6901 \times 10^{-6}$ & 13.7071 & & $3.2348 \times 10^{-8}$ & 17.4852 \\
12 & $4.8767 \times 10^{-8}$ & 19.4464 & & $3.7724 \times 10^{-10}$ & 24.4152 \\
\hline
\end{tabular}

we compare the absolute errors obtained using the MGLC method with $N=2, M=5, L=0.25$ and the method of [45]. 
Table 10: Absolute error obtained for Example 4.4 on $[0,1] \times[0,20]$ and $[0,1] \times[0,100]$ when $L=0.25$.

\begin{tabular}{llllll}
\hline$(\mathrm{t}, \mathrm{x})$ & $\begin{array}{l}\text { Yousefi et al. [45] } \\
(\mathrm{m}=5, \mathrm{n}=8)\end{array}$ & $\begin{array}{l}\text { MGLC (Case I) } \\
(\mathrm{N}=2, \mathrm{M}=5)\end{array}$ & $(\mathrm{t}, \mathrm{x})$ & Yousefi et al. [45] & MGLC (Case I) \\
\hline$(0,0)$ & 1.06 & 0.00 & $(0,0)$ & 3.21 & 0.00 \\
$(0.1,2)$ & $1.79 \times 10^{-1}$ & $2.42 \times 10^{-6}$ & $(0.1,10)$ & $8.93 \times 10^{-2}$ & $2.46 \times 10^{-12}$ \\
$(0.2,4)$ & $1.00 \times 10^{-1}$ & $2.08 \times 10^{-7}$ & $(0.2,20)$ & $5.47 \times 10^{-2}$ & $1.67 \times 10^{-20}$ \\
$(0.3,6)$ & $7.05 \times 10^{-4}$ & $4.66 \times 10^{-9}$ & $(0.3,30)$ & $1.03 \times 10^{-2}$ & $3.85 \times 10^{-29}$ \\
$(0.4,8)$ & $7.06 \times 10^{-2}$ & $8.18 \times 10^{-11}$ & $(0.4,40)$ & $3.06 \times 10^{-2}$ & $7.31 \times 10^{-38}$ \\
$(0.5,10)$ & $4.08 \times 10^{-2}$ & $2.47 \times 10^{-12}$ & $(0.5,50)$ & $2.29 \times 10^{-2}$ & $2.43 \times 10^{-46}$ \\
$(0.6,12)$ & $3.72 \times 10^{-2}$ & $6.75 \times 10^{-14}$ & $(0.6,60)$ & $1.48 \times 10^{-2}$ & $7.36 \times 10^{-55}$ \\
$(0.7,14)$ & $5.63 \times 10^{-2}$ & $6.54 \times 10^{-16}$ & $(0.7,70)$ & $2.75 \times 10^{-2}$ & $7.94 \times 10^{-64}$ \\
$(0.8,16)$ & $1.40 \times 10^{-2}$ & $9.41 \times 10^{-18}$ & $(0.8,80)$ & $8.61 \times 10^{-3}$ & $1.27 \times 10^{-72}$ \\
$(0.9,18)$ & $2.01 \times 10^{-2}$ & $1.66 \times 10^{-18}$ & $(0.9,90)$ & $8.17 \times 10^{-3}$ & $2.51 \times 10^{-80}$ \\
$(1,20)$ & $1.78 \times 10^{-1}$ & $2.43 \times 10^{-19}$ & $(1,100)$ & $8.45 \times 10^{-2}$ & $4.12 \times 10^{-88}$ \\
\hline
\end{tabular}

The absolute error function $\left|e_{M, N}(t, x)\right|$ with $T=2([0,2] \times[0,100]), N=2, L=0.25 M=14$ is shown in Figure 8.
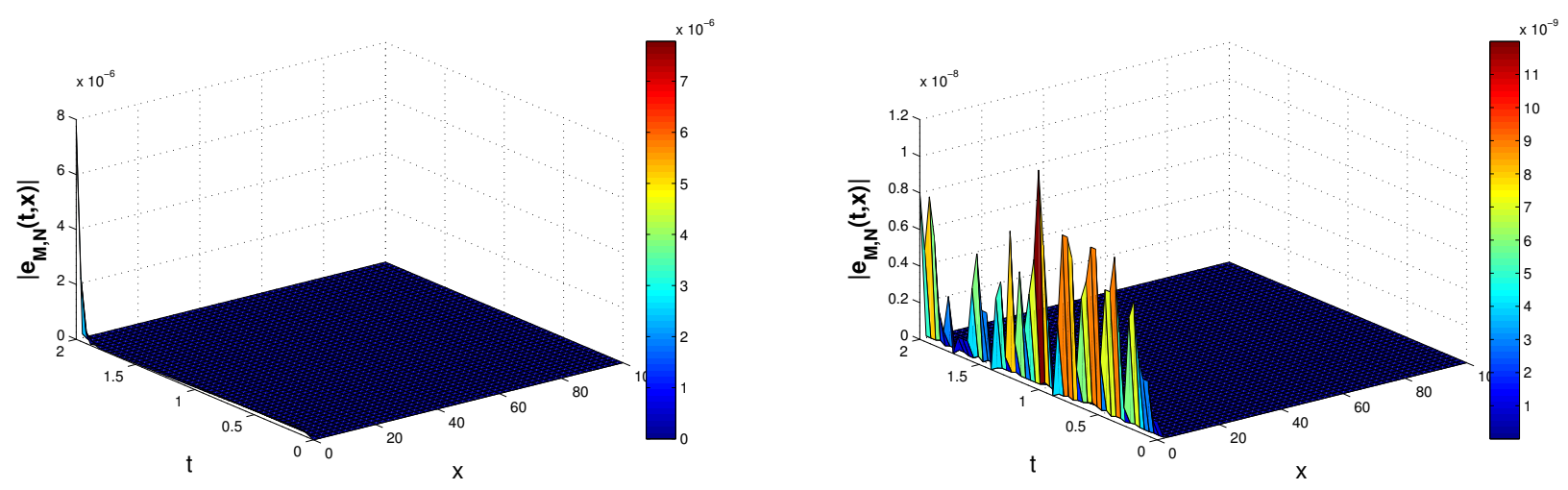

Figure 8: Absolute error function $\left|e_{M, N}(t, x)\right|$, for Case I (a) and Case II (b) where $M=14, N=2, T=2$ and $L=0.25$. See Example 4.4 .

Figures 9 and 12 (d) show the logarithmic of absolute values $u_{i j}$ as computed for case II with $\mathrm{T}=$ $1, N=5, M=6$ and the maximum absolute residual errors for case II with $T=1, N=2$ and various values of $L$, respectively. In this example, the optimum value of parameter $L$ is about $L=0.25$.

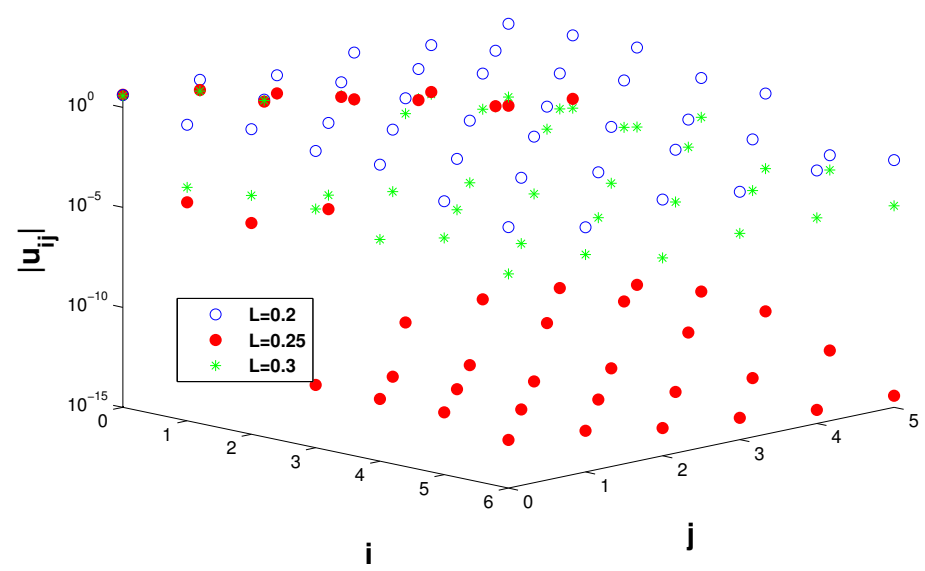

Figure 9: The logarithmic of absolute values of $u_{i j}$ versus $i$ and $j$ with $T=1, N=5, M=6$ and $L=0.2,0.25,0.3$ for Case II, Example 4.4. 
Example 4.5. Consider the following nonlinear age-structured population model [27]

$$
\begin{aligned}
\frac{\partial u(t, x)}{\partial t}+\frac{\partial u(t, x)}{\partial x} & =-\mu(x) u(t, x), \quad 0 \leqslant t, \quad 0 \leqslant x<A, \\
\mathfrak{u}(0, x) & =(1-x)^{\lambda} e^{-x}, \quad 0 \leqslant x<A, \\
\mathfrak{u}(t, 0) & =\int_{0}^{A} \beta(s) u(t, s) d s, \quad 0 \leqslant t,
\end{aligned}
$$

which $\mu(x)=\frac{\lambda}{1-x}$ and $A=+\infty$ and has the exact solution

$$
u(t, x)=(1-x)^{\lambda} e^{t-x}, \quad x \geqslant 0, \quad t \geqslant 0
$$

\begin{tabular}{|c|c|c|c|c|}
\hline \multirow[t]{2}{*}{$\bar{M}$} & \multicolumn{2}{|l|}{ Case I } & \multicolumn{2}{|l|}{ Case II } \\
\hline & $e_{M, N} \|_{\infty}$ & C-order & $\left\|e_{M, N}\right\|_{\infty}$ & C-order \\
\hline 4 & $4.3154 \times 10^{-4}$ & - & $1.3511 \times 10^{-4}$ & 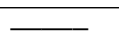 \\
\hline 6 & $1.5607 \times 10^{-6}$ & 13.8661 & $1.8090 \times 10^{-7}$ & 16.3168 \\
\hline 8 & $3.1803 \times 10^{-9}$ & 21.5374 & $1.5115 \times 10^{-10}$ & 24.6363 \\
\hline 10 & $4.1664 \times 10^{-12}$ & 29.7462 & $8.4168 \times 10^{-14}$ & 33.5802 \\
\hline 12 & $3.8064 \times 10^{-15}$ & 38.3834 & $3.3302 \times 10^{-17}$ & 42.9733 \\
\hline 14 & $2.5638 \times 10^{-18}$ & 47.3754 & $9.8269 \times 10^{-21}$ & 52.7292 \\
\hline
\end{tabular}

We approximate $u(t, x)$ on $[0,1] \times[0,100]$ and present the the maximum absolute values of errors and computational orders for $\mathrm{N}=3, \lambda=3, \mathrm{~L}=0.5$ and different values of $\mathrm{M}$ in Table 11.

Table 11: Errors and computational orders obtained for Example 4.5 with $T=1, \mathrm{~N}=3, \lambda=3$, and $\mathrm{L}=0.5$.

In Table 12, we compare the absolute errors obtained using the MGLC method with $\mathrm{N}=4, \mathrm{M}=8, \mathrm{~L}=$ $0.5, \lambda=3$, and the method of [45].

Table 12: Absolute error obtained for Example 4.5 for $\lambda=3$ on $[0,1] \times[0,20]$ and $[0,1] \times[0,100]$ when $L=0.5$.

\begin{tabular}{llllll}
\hline$(\mathrm{t}, \mathrm{x})$ & $\begin{array}{l}\text { Yousefi et al. [45] } \\
(\mathrm{m}=5, \mathrm{n}=10)\end{array}$ & $\begin{array}{l}\text { MGLC (Case I) } \\
(\mathrm{N}=4, \mathrm{M}=8)\end{array}$ & $(\mathrm{t}, \mathrm{x})$ & $\begin{array}{l}\text { Yousefi et al. [45] } \\
(\mathrm{m}=5, \mathrm{n}=10)\end{array}$ & $\begin{array}{l}\text { MGLC (Case I) } \\
(\mathrm{N}=4, \mathrm{M}=8)\end{array}$ \\
\hline$(0,0)$ & $2.90 \times 10^{-1}$ & 0.00 & $(0,0)$ & $5.59 \times 10^{-1}$ & 0.00 \\
$(0.1,2)$ & $2.31 \times 10^{-2}$ & $1.38 \times 10^{-11}$ & $(0.1,10)$ & $2.44 \times 10^{-2}$ & $3.61 \times 10^{-12}$ \\
$(0.2,4)$ & $1.26 \times 10^{-1}$ & $5.35 \times 10^{-11}$ & $(0.2,20)$ & $1.16 \times 10^{-1}$ & $1.53 \times 10^{-15}$ \\
$(0.3,6)$ & $8.40 \times 10^{-2}$ & $3.63 \times 10^{-11}$ & $(0.3,30)$ & $9.19 \times 10^{-2}$ & $2.67 \times 10^{-19}$ \\
$(0.4,8)$ & $8.30 \times 10^{-2}$ & $1.56 \times 10^{-11}$ & $(0.4,40)$ & $2.47 \times 10^{-2}$ & $3.41 \times 10^{-23}$ \\
$(0.5,10)$ & $7.26 \times 10^{-3}$ & $4.70 \times 10^{-12}$ & $(0.5,50)$ & $2.96 \times 10^{-1}$ & $3.22 \times 10^{-27}$ \\
$(0.6,12)$ & $1.46 \times 10^{-3}$ & $1.36 \times 10^{-12}$ & $(0.6,60)$ & $5.32 \times 10^{-1}$ & $2.99 \times 10^{-31}$ \\
$(0.7,14)$ & $1.17 \times 10^{-2}$ & $3.14 \times 10^{-13}$ & $(0.7,70)$ & $1.97 \times 10^{-1}$ & $2.25 \times 10^{-35}$ \\
$(0.8,16)$ & $1.92 \times 10^{-2}$ & $7.65 \times 10^{-14}$ & $(0.8,80)$ & $8.81 \times 10^{-1}$ & $1.79 \times 10^{-39}$ \\
$(0.9,18)$ & $2.10 \times 10^{-2}$ & $2.05 \times 10^{-14}$ & $(0.9,90)$ & 1.35 & $1.58 \times 10^{-43}$ \\
$(1,20)$ & $6.46 \times 10^{-2}$ & $4.50 \times 10^{-14}$ & $(1,100)$ & 3.46 & $1.15 \times 10^{-46}$ \\
\hline
\end{tabular}

The absolute error function $\left|e_{M, N}(t, x)\right|$ with $T=5([0,5] \times[0,100]), N=4, \lambda=3, L=0.5$ and $M=20$ is shown in Figure 10. Figure 11 depicts the logarithmic of absolute values $u_{i j}$ as computed for case II with $T=1, \lambda=3, N=8, M=16$ and several scaling factor $L$. We observe that the optimum value of parameter $\mathrm{L}$ is about $\mathrm{L}=0.5$.

\section{Conclusion}

In this study, we investigated the verification of approximation method for solving the age-structured population model in different magnitude of domain by using MGLC method.

As a disadvantage of the Laguerre method, we can mention to the non-applicability. However, the Laguerre polynomials are orthogonal on $[0, \infty)$, the collocation method based on these functions practically 
is not efficient. In fact, programming clearly shows that the theoretical results are not achievable. This paper focuses on some modifications which can highly improve the rate of accuracy to the exponential level. In fact, the spectral accuracy is considered as a very important advantage in numerical approximation methods due to decreasing the cost of computations considerably. Note that finite element method (FEM) and finite different method (FDM) are not as fast as MGLC method. The other advantage of this method is simplicity in unifying the boundary condition and the equation under study. We remind that, age-structured population model is associated with an integral boundary condition which causes very complicated computations. Actually, in some methods such as FEM, FDM, and etc. dealing with boundary conditions is a costly challenge but MGLC method is flexible respect with boundary conditions.

Theoretical analysis and experimental results show the validity, accuracy, and computational efficiency of the MGLC method. The numerical experiment for different $A$ and T shows the validity of the method even in extended areas. As such the method is reliable in the larger scale problems if we choose the right kind of orthogonal polynomials for the approximation. Furthermore, according to the the results of this research if population model is proposed for long-term simulation, we can change the structure of approximated solution based on Laguerre-Laguerre spectral collocation method, which means two direction may be approximated by the modified generalized Laguerre functions. We emphasize the modification of generalized Laguerre functions provides not only the optimum rate convergence but the method also considers the size of area and behavior of the solution as $x$ approaches infinity.

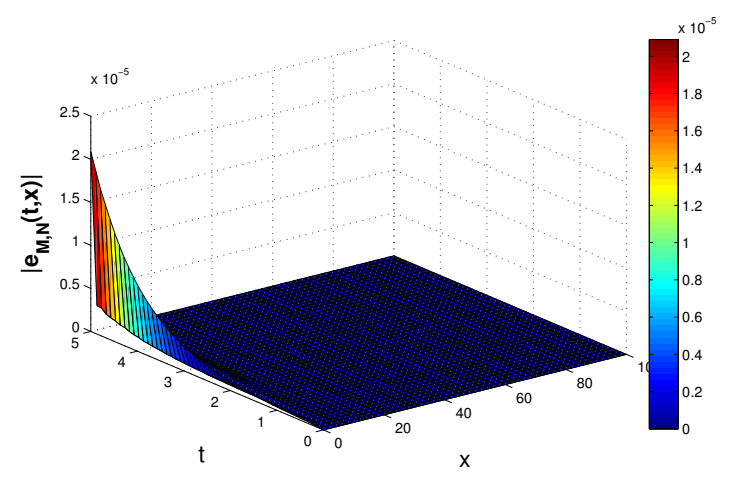

(a)

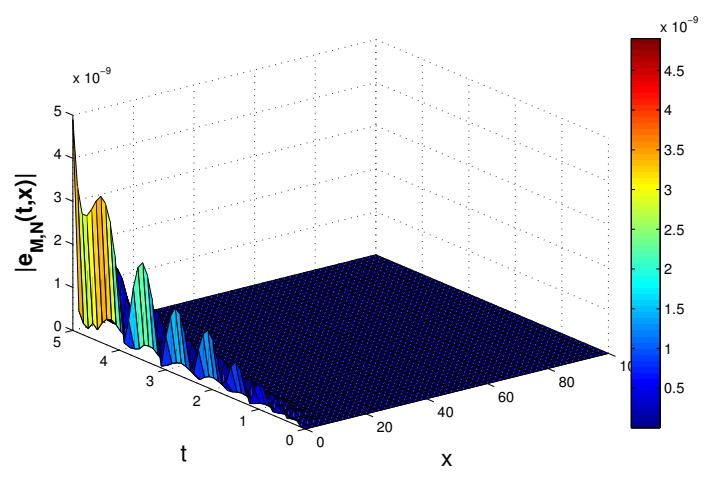

(b)

Figure 10: Absolute error function $\left|e_{M, N}(t, x)\right|$, for Case I (a) and Case II (b), where $M=20, N=4, T=5, \lambda=3$, and $L=0.5$. See Example 4.5.

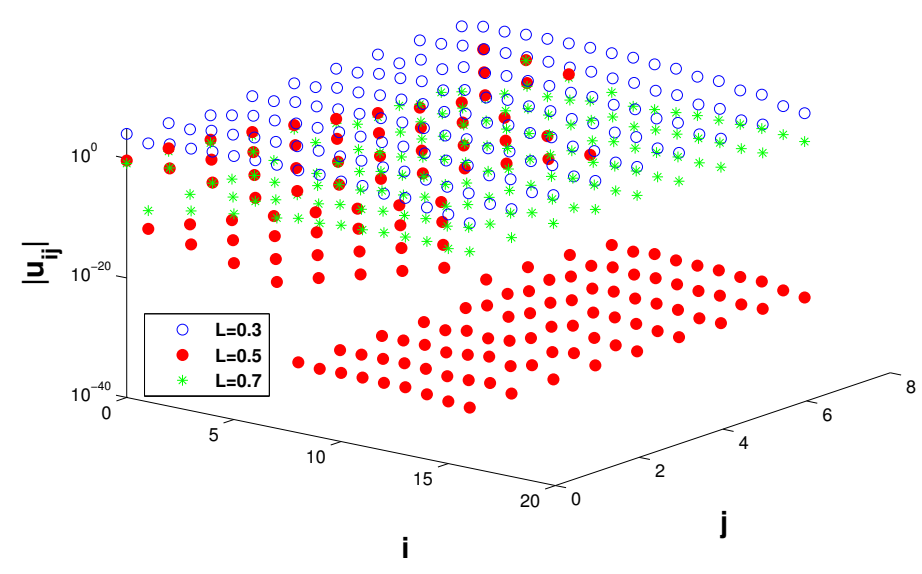

Figure 11: The logarithmic of absolute values of $u_{i j}$ versus $i$ and $j$ with $T=1, \lambda=3, N=8, M=16$, and $L=0.3,0.5,0.7$ for Case II, Example 4.5. 
(a)

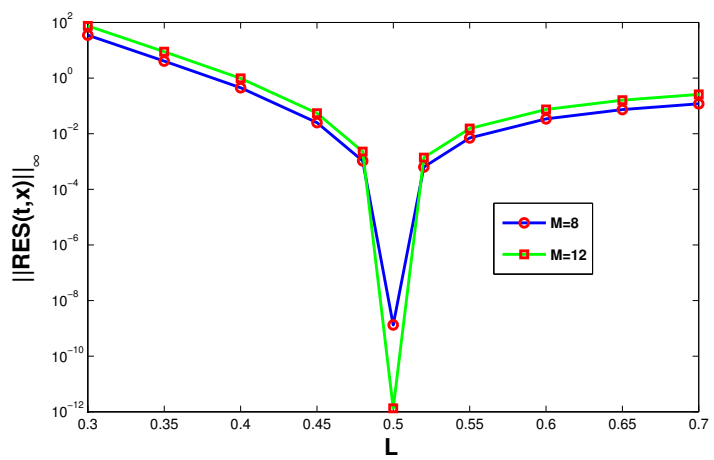

(c)

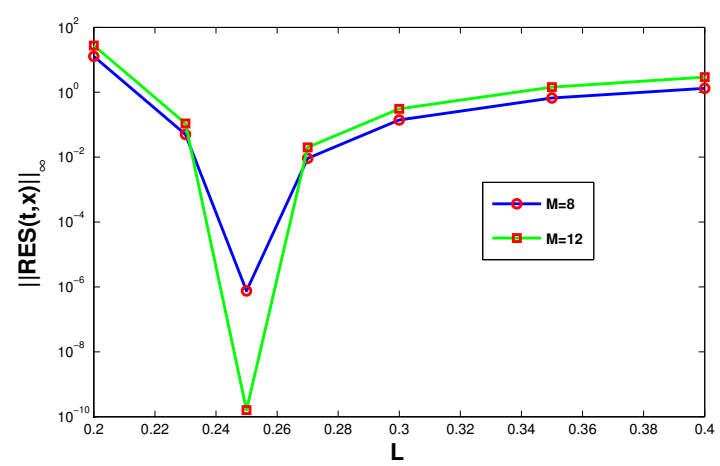

(b)

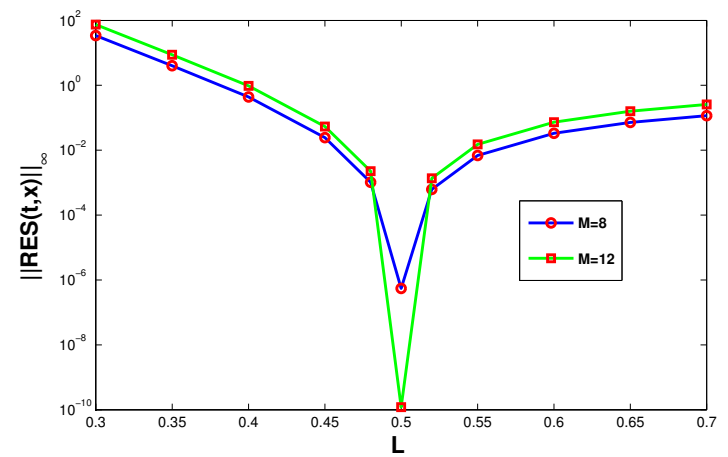

(d)

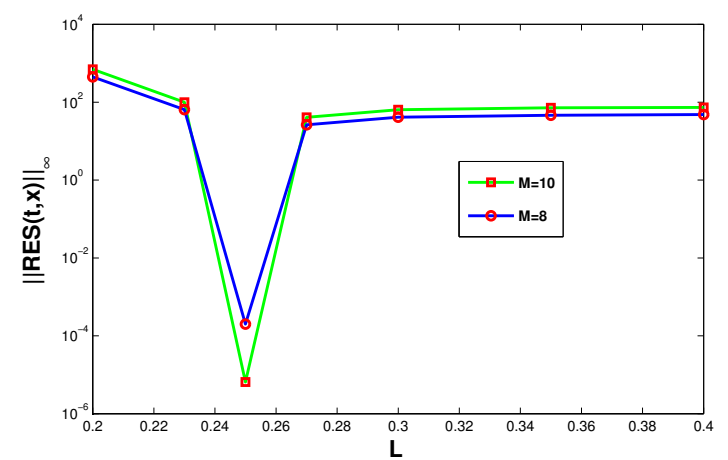

Figure 12: Comparison of the maximum absolute residual errors for $u(t, x)$ with different choices of the parameter $L$ for Case II in Examples 4.1, 4.2, 4.3, and 4.4 depicted in (a), (b), (c), and (d), respectively, where $\mathrm{N}=2$ and $\mathrm{T}=1$.

\section{Acknowledgment}

Zakieh Avazzadeh wishes to thank Natural Science Foundation of Jiangsu Province (Grant No. BK20150964).

\section{References}

[1] S. Abbasbandy, E. Shivanian, Numerical simulation based on meshless technique to study the biological population model, Math. Sci., 10 (2016), 123-130. 1

[2] L. M. Abia, O. Angulo, J. C. López-Marcos, Age-structured population dynamics models and their numerical solutions, Ecological Modelling, 188 (2005), 112-136. 1, 1

[3] L. M. Abia, O. Angulo, J. C. López-Marcos, M. A. López-Marcos, Numerical schemes for a size-structured cell population model with equal fission, Math. Comput. Modelling, 50 (2009), 653-664. 1

[4] L. M. Abia, J. C. López-Marcos, Second order schemes for age-structured population equations, J. Biol. Syst., 5 (1997), $1-16$.

[5] O. Angulo, J. C. López-Marcos, M. A. López-Marcos, F. A. Milner, A numerical method for nonlinear age-structured population models with finite maximum age, J. Math. Anal. Appl., 361 (2010), 150-160. 1, 4.3, 4.4

[6] Z. Avazzadeh, M. Heydari, Chebyshev polynomials for solving two dimensional linear and nonlinear integral equations of the second kind, Comput. Appl. Math., 31 (2012), 127-142. 2

[7] Z. Avazzadeh, M. Heydari, Chebyshev cardinal functions for solving age-structured population models, Int. J. Appl. Comput. Math., 3 (2017), 2139-2149. 1

[8] Z. Avazzadeh, M. Heydari, Haar wavelet method for solving nonlinear age-structured population models, Int. J. Biomath., 10 (2017), 21 pages. 1

[9] S. S. Bayin, Mathematical Methods in Science and Engineering, John Wiley \& Sons, Hoboken, (2006). 2.2

[10] G. Ben-yu, W. Zhong-qing, Numerical integration based on Laguerre-Gauss interpolation, Comput. Methods Appl. Mech. Engrg., 196 (2007), 3726-3741. 1

[11] B. Blasius, J. R. Kurths, L. Stone, Complex Population Dynamics, World Scientific, New York, (2007). 1 
[12] J. P. Boyd, The optimization of convergence for Chebyshev polynomial methods in an unbounded domain, J. Comput. Phys., 45 (1982), 43-79. 2.4, 4

[13] J. P. Boyd, Spectral methods using rational basis functions on an infinite interval, J. Comput. Phys., 69 (1987), 112-142. 4

[14] J. P. Boyd, The rate of convergence of Fourier coefficients for entire functions of infinite order with application to the Weideman-Cloot sinh-Mapping for pseudospectral computations on an infinite interval, J. Comput. Phys., 110 (1994), 360-372.

[15] J. P. Boyd, Chebyshev and Fourier Spectral Methods, Dover Publication Inc., Mineola, (2001). 1, 2, 2.1, $2.4,4$

[16] C. Canuto, M. Y. Hussaini, A. Quarteroni, T. A. Zang, Spectral Methods in Fluid Dynamics, Springer-Verlag, New York, (1988). 1, 2, 2.1, 2.1, 3

[17] M. G. Cui, Z. Chen, The exact solution of nonlinear age-structured population model, Nonlinear Anal. Real World Appl., 8 (2007), 1096-1112. 1, 4.1

[18] C. Cusulin, L. Gerardo-Giorda, A numerical method for spatial diffusion in age-structured populations, Numer. Methods Partial Differential Equations, 26 (2010), 253-273. 1

[19] P. J. Davis, Interpolation and Approximation, Dover Publications, New York, (1975). 2.2.1

[20] D. Funaro, Polynomial Approximation of Differential Equations, Springer-Verlag, Berlin, (1992). 2.2

[21] M. Ghoreishi, A. I. B. M. Ismail, A. K. Alomari, A. S. Bataineh, The comparison between homotopy analysis method and optimal homotopy asymptotic method for nonlinear age-structured population models, Commun. Nonlinear Sci. Numer. Simul., 17 (2012), 1163-1177. 1, 3, 6

[22] M. Heydari, S. M. Hosseini, G. B. Loghmani, Numerical solution of singular IVPs of Lane-Emden type using integral operator and radial basis functions, Int. J. Ind. Math., 4 (2012), 135-146. 1

[23] M. Heydari, G. B. Loghmani, A. A. Dehghan, A combination of pseudo-spectral method and extrapolation for solving MHD flow and heat transfer about a rotating disk, Iran. J. Sci. Technol. Trans., 38 (2014), 25-44. 2.4

[24] M. Heydari, G. B. Loghmani, A. A. Dehghan, Numerical study of generalized three-dimensional MHD flow over a porous stretching sheet, J. Appl. Fluid. Mech., 7 (2014), 473-483. 1

[25] M. M. Hosseini, S. T. Mohyud-Dinb, H. Ghaneai, Variational iteration method for nonlinear age-structured population models using auxiliary parameter, Z. Naturforsch. A, 65 (2010), 1137-1142. 1

[26] M. Iannelli, M.-Y. Kim, E.-J. Park, Splitting method for the numerical approximation of some models of age-structured population dynamics and epidemiology, Appl. Math. Comput., 87 (1997), 69-93. 1

[27] M. Iannelli, F. A. Milner, On the approximation of the Lotka-McKendrick equation with finite life-span, J. Comput. Appl. Math., 136 (2001), 245-254. 4.5

[28] A. Kadem, D. Baleanu, Analytical method based on Walsh function combined with orthogonal polynomial for fractional transport equation, Commun. Nonlinear. Sci. Numer. Simul., 15 (2010), 491-501. 2

[29] A. Kadem, D. Baleanu, Fractional radiative transfer equation within Chebyshev spectral approach, Comput. Math. Appl., 59 (2010), 1865-1873. 2

[30] F. Kappel, K. P. Zhang, Approximation of linear age-structured population models using Legendre polynomials, J. Math. Anal. Appl., 180 (1993), 518-549. 1

[31] M.-Y. Kim, E.-J. Park, An upwind scheme for a nonlinear model in age-structured population dynamics, Comput. Math. Appl., 30 (1995), 5-17. 1

[32] H. Koçak, A. Yildirim, An efficient algorithm for solving nonlinear age-structured population models by combining homotopy perturbation and Padé techniques, Int. J. Comput. Math., 88 (2011), 491-500. 1, 4.1, 4.2

[33] P. Krzyżanowski, D. Wrzosek, D. Wit, Discontinuous Galerkin method for piecewise regular solution to the nonlinear age-structured population model, Math. Biosci., 203 (2006), 277-300. 1

[34] P. K. Kythe, M. R. Schäferkotter, Handbook of Computational Method for Integration, Chapman \& Hall/CRC Press, Boca Raton, (2005). 2

[35] X. Y. Li, Variational iteration method for nonlinear age-structured population models, Comput. Math. Appl., 58 (2009), 2177-2181. 1, 4.1, 4.2

[36] Z. Nikooeinejad, A. Delavarkhalafi, M. Heydari, A numerical solution of open-loop Nash equilibrium in nonlinear differential games based on Chebyshev pseudospectral method, J. Comput. Appl. Math., 300 (2016), 369-384. 2

[37] Z. Nikooeinejad, A. Delavarkhalafi, M. Heydari, Application of shifted Jacobi pseudospectral method for solving (in) finite-horizon minmax optimal control problems with uncertainty, Internat. J. Control, 13 (2017), 725-739.

[38] Z. Nikooeinejad, M. Heydari, Nash equilibrium approximation of some class of stochastic differential games: A combined Chebyshev spectral collocation method with policy iteration, J. Comput. Appl. Math., 362 (2019), 41-54. 2

[39] K. Parand, A. R. Rezaei, A. Taghavi, Lagrangian method for solving Lane-Emden type equation arising in astrophysics on semi-infinite domains, Acta Astronautica, 67 (2010), 673-680. 2.2, 2.2.2

[40] J. Shen, Stable and efficient spectral methods in unbounded domains using Laguerre functions, SIAM J. Numer. Anal., 38 (2000), 1113-1133. 1

[41] J. Shen, T. Tang, L.-L. Wang, Spectral Methods: Algorithms, Analysis and Applications, Springer-Verlag, Berlin, (2011). $1,2,2.2,2.2 .1,2.2 .2$

[42] G. Szegö, Orthogonal Polynomials, American Mathematical Society, Providence, (1975). 2.3

[43] M. Tatari, M. Haghighi, A generalized Laguerre-Legendre spectral collocation method for solving initial-boundary value problems, Appl. Math. Model., 38 (2014), 1351-1364. 1 
[44] J. Valenciano, M. A. J. Chaplain, A Laguerre-Legendre spectral-element method for the solution of partial differential equations on infinite domains: Application to the diffusion of tumour angiogenesis factors, Math. Comput. Modelling, 41 (2005), 1171-1192. 1

[45] S. A. Yousefi, M. Behroozifar, M. Dehghan, Numerical solution of the nonlinear age-structured population models by using the operational matrices of Bernstein polynomials, Appl. Math. Model., 36 (2012), 945-963. 1, 4.1, 4, 2, 4.2, 4, 5, $4,8,4,10,4,12$

[46] L. W. Zhang, Y. J. Deng, K. M. Liew, An improved element-free Galerkin method for numerical modeling of the biological population problems, Eng. Anal. Bound. Elem., 40 (2014), 181-188. 1 